\title{
Robo2-Slit and Dcc-Netrin1 Coordinate Neuron Axonal Pathfinding within the Embryonic Axon Tracts
}

\author{
Changwen Zhang, ${ }^{\star}$ Jingxia Gao, ${ }^{\star}$ Hefei Zhang, Liu Sun, and Gang Peng \\ Institutes of Brain Science and State Key Laboratory of Medical Neurobiology, Fudan University, Shanghai 200032, China
}

In the embryonic vertebrate brain, early born neurons establish highly stereotyped embryonic axonal tracts along which the neuronal interconnections form. To understand the mechanism underlying neuron axonal pathfinding within the embryonic scaffold of axon tracts, we studied zebrafish anterior dorsal telencephalic (ADt) neuron development. While previous studies suggest the ADt neuronal axons extend along a commissural tract [anterior commissure (AC)] and a descending ipsilateral tract [supraoptic tract (SOT)], it is unclear whether individual ADt neuronal axons choose specific projection paths at the intersection between the AC and the SOT. We labeled individual ADt neurons using a forebrain-specific promoter to drive expression of fluorescent proteins. We found the ADt axonal projection patterns were heterogeneous and correlated with their soma positions. Our results suggest that cell intrinsic differences along the dorsal ventral axis of the telencephalon regulate the axonal projection choices. Next, we determined that the guidance receptors roundabout2 (Robo2) and deleted in colorectal cancer (Dcc) were differentially expressed in the ADt neurons. We showed that knocking down Robo2 function by injecting antisense morpholino oligonucleotides abolished the ipsilateral SOT originating from the ADt neurons. Knocking down Dcc function did not prevent formation of the AC and the SOT. In contrast, the AC was specifically reduced when Netrin1 function was knocked down. Further mechanistic studies suggested that Robo2 responded to the repellent Slit signals and suppressed the attractive Netrin signals. These findings demonstrate how Robo2-Slit and Dcc-Netrin coordinate the axonal projection choices of the developing neurons in the vertebrate forebrain.

\section{Introduction}

The vertebrate brain contains many distinct types of neurons. Multiple transcription factors and guidance signals are needed for the specification and axonal pathfinding of the diverse neuronal populations (Polleux et al., 2007; Molyneaux et al., 2007). Groups of different types of neurons often form discrete nuclei and project axonal tracts over long distance to specific regions of the brain. The organization of vertebrate brain into nuclei and axonal tracts has its foundation in the embryonic development of the brain (Chédotal and Richards, 2010). In the embryonic brain, neurons are specified as clusters in different regions of the brain. Axons originating from these neuronal clusters migrate in defined directions and establish highly stereotyped embryonic scaffold of axonal tracts (Chitnis and Kuwada, 1990; Wilson et al., 1990; Easter et al., 1993, 1994). Growth cones of these neurons are known to choose their appropriate projection pathways at the intersection between different axonal tracts (Chitnis and $\mathrm{Ku}$ -

Received Dec. 30, 2011; revised July 10, 2012; accepted July 20, 2012.

Author contributions: C.Z., J.G., and G.P. designed research; C.Z., J.G., H.Z., L.S., and G.P. performed research; C.Z., J.G., and G.P. analyzed data; G.P. wrote the paper.

This work was supported by the National 985 Program sponsored by the Ministry of Education of China, National Natural Science Foundation of China (Grants 30900861, 30970948), the Pujiang Talent Project (Grant 09PJ1401900), and the Innovation Program of Shanghai Education Commission (Grant 11ZZ05). We thank M. Westerfield for advice and support. We thank C. Zhang, L. Shen, and L. Gao for fish care and Y. Shi, M. Zhou, and Q. Huang for technical assistance.

${ }^{*}$ C.Z. and J.G. contributed equally to this work.

Correspondence should be addressed to Gang Peng, Institutes of Brain Science, Fudan University, 138 Yi Xue Yuan Road, Shanghai 200032, China. E-mail: gangpeng@fudan.edu.cn.

DOI:10.1523/JNEUROSCI.6518-11.2012

Copyright $\odot 2012$ the authors $\quad 0270-6474 / 12 / 3212589-14 \$ 15.00 / 0$ wada, 1990). Transplantation experiments, ablation studies, and mutation analyses have shown that axonal pathfinding within the embryonic scaffold of axonal tracts are highly regulated (Chitnis and Kuwada, 1991; Patel et al., 1994; Kanki and Kuwada, 2000).

In the embryonic zebrafish and Xenopus forebrains, the telencephalic neurons extend axons along a commissural tract [anterior commissure $(\mathrm{AC})]$ and a descending ipsilateral tract [telencephalon tract or supraoptic tract (SOT)] by the pharyngula period (Chitnis and Kuwada, 1990; Wilson et al., 1990; Anderson and Key, 1996). Multiple guidance signals are involved in the formation of the AC and the SOT (Wolman et al., 2004; Wilson and Key, 2006). However, few studies have examined the heterogeneity of the telencephalic neurons, and it is unclear whether the individual axons choose specific projection paths at the intersection between the $\mathrm{AC}$ and the SOT. To understand the mechanism underlying neuron axonal pathfinding within the embryonic scaffold of axon tracts, we studied zebrafish anterior dorsal telencephalic (ADt) neuron development. We examined the axonal projection pattern of individual ADt neurons by labeling the cell using a forebrain-specific promoter to drive expression of fluorescent proteins. Our results suggested that the ADt neurons were composed of distinct population of projection neurons and cell intrinsic differences may regulate the development of the ADt neurons. We next determined that the guidance receptors roundabout2 (Robo2) and deleted in colorectal cancer (Dcc) were differentially expressed in the ADt neurons. We showed that knocking down Robo2 function by injecting antisense morpholino (MO) oligonucleotides abolished the descending SOT labeled by fluorescent proteins in transgenic zebrafish 
embryos. Whereas knocking down Dcc function did not affect the AC and the SOT formation, the AC was specifically reduced when Netrin 1 function was knocked down. Further mechanistic studies showed that Robo2 responded to the repellent Slit signal and suppressed the attractive Netrin 1 signal. These findings demonstrate how Robo2-Slit and Dcc-Netrin signaling pathways coordinate the axonal projection choices of the developing neurons in the vertebrate forebrain.

\section{Materials and Methods}

Fish maintenance. Zebrafish were maintained in a recirculating water system according to standard protocol (Westerfield, 2007). Embryos were staged by hours postfertilization (hpf) as described previously (Kimmel et al., 1995). The Fudan University Institutional Animal Care and Use Committee approved all work with zebrafish animals.

Morpholino oligonucleotides and synthetic mRNA. Morpholinos were synthesized by Gene Tools: standard control, 5'-CCTCTTACCTCAGTTACA ATTTATA (4 ng or 6 ng); dcc-MO1, 5' -GAATATCTC CAGTGACGCAGC CCAT [ 6 ng; described previously by Suli et al. (2006)]; dcc-MO2, 5' -GCGA AATCCGCTGCTAATCAATCAA [ $6 \mathrm{ng}$; described previously by Suli et al. (2006)]; dcc-MO3, 5'-GAGCAGCACTGACCGTGTGTGTAGA [4 ng; described previously by Suli et al. (2006)]; ntn1a-MO, $5^{\prime}$-CCAAAGCATCAG AGACTCTCAACAT (4 ng); ntn1b-MO, 5'-CGCACGTTACCAAAATCC TTATCAT [4 ng; described previously by Suli et al. (2006)]; robo2 translation blocking MO (robo2-tMO), 5'-AAGGACCCATCCTGTCATAG TCCAC (4 ng); robo2 splice blocking MO (robo2-sMO), GTAGCGCAAC TCACCATCACTTGG (4 ng); slit1a-MO, 5' -GAAATAAACTCACAGCCT CTCGGTG [ $2 \mathrm{ng}$; described previously by Zolessi et al. (2006) and Campbell et al. (2007)]; slit1b-MO, GCTCGGTGTCCGGCATCTCCAAAAG [4 ng; described previously by Zolessi et al. (2006) and Kastenhuber et al. (2009)]; slit2-MO, CATCACCGCTGTTTCCTCAAGTTCT [2 ng; described previously by Barresi et al. (2005) and Kastenhuber et al. (2009)]; slit3-MO, TA TATCCTCTGAGGCTGATAGCAGC [2 ng; described previously by Barresi et al. (2005) and Kastenhuber et al. (2009)]. The sources for morpholino descriptions and the dosages for morpholino injections (per embryo) were indicated in parentheses.

Primers used in RT-PCR analysis of robo2-sMO effects were as follows: ZW30, 5' -TCAAAGAGGAGAAACGTGTTCTGG (robo2 forward primer); ZW31, 5'-GTGGGATCGAGGGTCATCTTTG (robo2 reverse primer). The primers used for housekeeping gene odcl were described previously by Peng and Westerfield (2006).

Zebrafish robo2 cDNAs were amplified by RT-PCR with the following primers: ZW43, 5'-GGGTTGAGAACTGAGGTGTGGATGTGGAC (robo2 cDNA forward); ZW44, 5'-ACTGCACCAAGCAGGTGAGGCATTTCC $\mathrm{AG}$ (robo $2 \mathrm{cDNA}$ reverse). To construct a robo $2 \mathrm{cDNA}$ that was resistant to morpholino antisense blocking effects (robo2-R mRNA), silent mutations were introduced into the robo2 cDNA sequence with forward primer robo2-

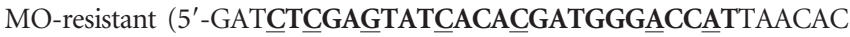
ACCTTTTACTCTGTGG; the morpholino targeting site is in bold and the mutated nucleotides are underlined). Silent mutations were introduced into the $d c c$ cDNA sequence with primer $d c c$-MO1-resistant (5'-CTCAAGCTTC GAATTCTCCACCATGGGGTGTGTGACAGGTGACATACGCCGACTT TCCGCGCTCCTC) using a full length $d c c$ cDNA clone as PCR template (the morpholino targeting site is in bold and the mutated nucleotides are underlined). Capped mRNA was synthesized with the mMESSAGE mMACHINE kit (Ambion). The dosage for injection was $250 \mathrm{pg}$ of synthetic robo2 or $350 \mathrm{pg}$ of synthetic $d c c$ mRNA per embryo.

Genetic single-cell labeling. A mixture of two plasmids -5.2emx3: Gal4FF and $5 \times U A S: E G F P$ or $5 \times U A S: t d T o m a t o(10 \mathrm{ng} / \mu \mathrm{l}$ each) was injected into one-cell-stage embryos. Injected embryos were screened by fluorescent dissection microscopy at $24 \mathrm{hpf}$ and then by confocal microscopy at 36 hpf to identify animals with axons that could be traced and assigned to labeled single cells. To label cells in robo2-tMO- or $d c c-\mathrm{MO}-$ injected animals, robo2-tMO or $d c c-\mathrm{MO}$ morpholino was injected at the one- to four-cell stage after the plasmid injection.

Whole-mount in situ hybridization. Fluorescent in situ hybridization was performed as described previously (Talbot et al., 2010). Digoxigenin-labeled $d c c$ and fluorescein-labeled robo2 antisense RNA probes were detected by
Tyramide Signal Amplification kits (Invitrogen). The $d c c$ clone was described previously (Suli et al., 2006). To make the robo2 clone, RT-PCR was performed with primers robo2 forward (5'-CAAGATGAATGGCTCCAAC TGGGC) and robo2 reverse (5'-AGCTGCTTTCACCTTGTTCCGTGG). Whole-mount in situ hybridization was performed as described previously (Peng and Westerfield, 2006). Digoxigenin-labeled antisense RNA probes were detected with alkaline phosphatase-conjugated digoxigenin antibody Fab fragment (1:7500; Roche) and alkaline phosphatase substrate NBT/ BCIP (1:80; Roche). The Netrin 1 clones used in this study were as follows: $n t n 1 a$ (GenBank accession number, BC114259) and ntn1b (GenBank accession number, CK684895). To make the Slit clones, RT-PCRs were performed with primers slit1a forward (5'-GACTGC CAGAACAACGCCCC AT), slit1 a reverse (5'-GGTTTTCTCCACCTCCTCGCTG), slit $1 b$ forward (5' -CTGCTGCTGAGAGATGCTACACAAT), slit $1 b$ reverse (5' -CTTTCC ACAGCCA CACTCCACTG), slit2 forward (5'-CCGATGGTCCTGCCAA GAACAAG), slit2 reverse (5'-GATGGACATTTTGTGCAGCCACAC), slit3 forward ( 5 '-CTGGTTACTTCGGCACTAA GTGTC), and slit3 reverse (5'-GCAGCCACACTCCACAGTGATC). All clones were verified by sequencing.

Photoconversion of Kaede. Photoconversion of Kaede was performed with a laser scanning confocal microscope (Olympus FV1000) equipped with a $405 \mathrm{~nm}$ laser. Live embryos were mounted with $1.2 \%$ low-meltingtemperature agarose, and the ADt region on one hemisphere of the telencephalon was marked with the polygon selection tool of the FluoView software (Olympus). Ten pulses of focused $405 \mathrm{~nm}$ laser light were then applied to the selected region. Each pulse lasted $500 \mathrm{~ms}$ and used $60 \%$ of the light output of the $405 \mathrm{~nm}$ laser. Confocal image stacks were acquired $30 \mathrm{~min}$ later after the converted Kaede diffused into the neuronal processes.

Whole-mount antibody staining. To stain Kaede and acetylate tubulin, fish embryos were fixed with $2 \%$ trichloroacetic acid $(\mathrm{w} / \mathrm{v})$ in $0.1 \%$ Tween-20 in PBS at room temperature for $1 \mathrm{~h}$. Fixed embryos were blocked with $1 \times$ blocking solution (Roche) at room temperature for $3 \mathrm{~h}$. Blocked embryos were incubated with acetylated tubulin antibodies (1: 800; Sigma) and Kaede antibodies (1:100; MBL International) in $1 \times$ blocking solution at $4^{\circ} \mathrm{C}$ overnight, followed by incubation with Alexa 488- and Alexa 546-conjugated secondary antibodies (1:100; Invitrogen) at room temperature for $3 \mathrm{~h}$. To stain tdTomato-labeled cells, postfluorescent in situ hybridization embryos were incubated with anti-DsRed antibodies (1:100; Clontech) followed by incubation with Alexa 635conjugated secondary antibodies (1:100; Invitrogen).

Microscopy and image analysis. For confocal microscopy, live or fixed embryos were mounted with $1.2 \%$ low-melting-temperature agarose and imaged on an Olympus FV1000 confocal laser scanning system with a $40 \times$ water-immersion objective. For fluorescence in situ results, image stack projections or single image slices were exported from the FluoView software (Olympus). For other results, the captured image stacks were converted by ImageJ for reconstruction with FluoRender software (http://www.sci.utah.edu/software.html). Reconstructed three-dimensional images were projected to standardized views for figure presentation. To register images to common reference space, manually segmented images were automatically processed by custom written MATLAB scripts (MathWorks; scripts available upon request). The image registration method was based on a nonrigid B-spline function (Klein et al., 2011). To quantify the expression level of $d c c$ and robo2, the confocal data were batch converted by ImageJ Macro and then batch processed by MATLAB scripts (MathWorks). The MATLAB scripts used manually segmented cell body masks to index the $d c c$ and robo2 image channels and calculated the sum of the pixel intensity values corresponding to the indexed cell body positions (macros and scripts available upon request). To quantify the SOT/AC ratio, regions $(20 \times 40$ pixels $)$ of the SOT or the AC at locations proximate to the SOT-AC junction were selected (see Fig. $5 G$, boxes) and then batch processed by MATLAB scripts (MathWorks). The MATLAB scripts used automated thresholding methods to index the selected axon images and calculated the sum of the pixel intensity values corresponding to the indexed axonal positions (scripts available upon request). Statistical tests were performed with SPSS software. 


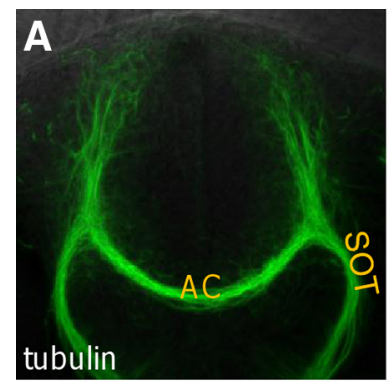

B
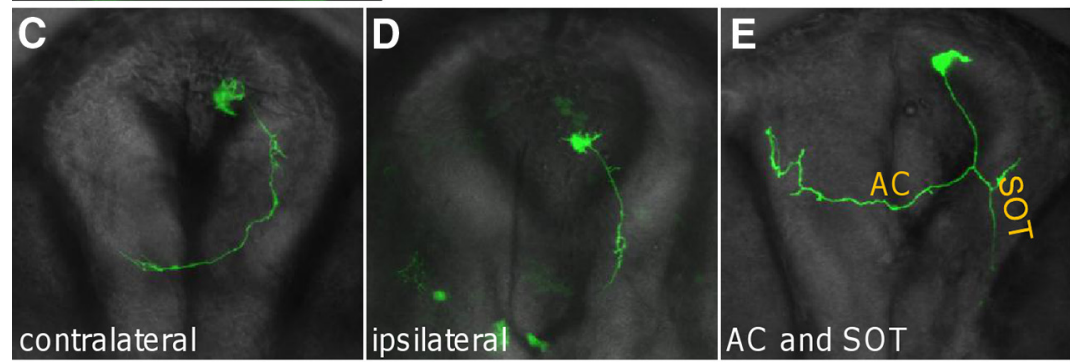

$\mathbf{F}$
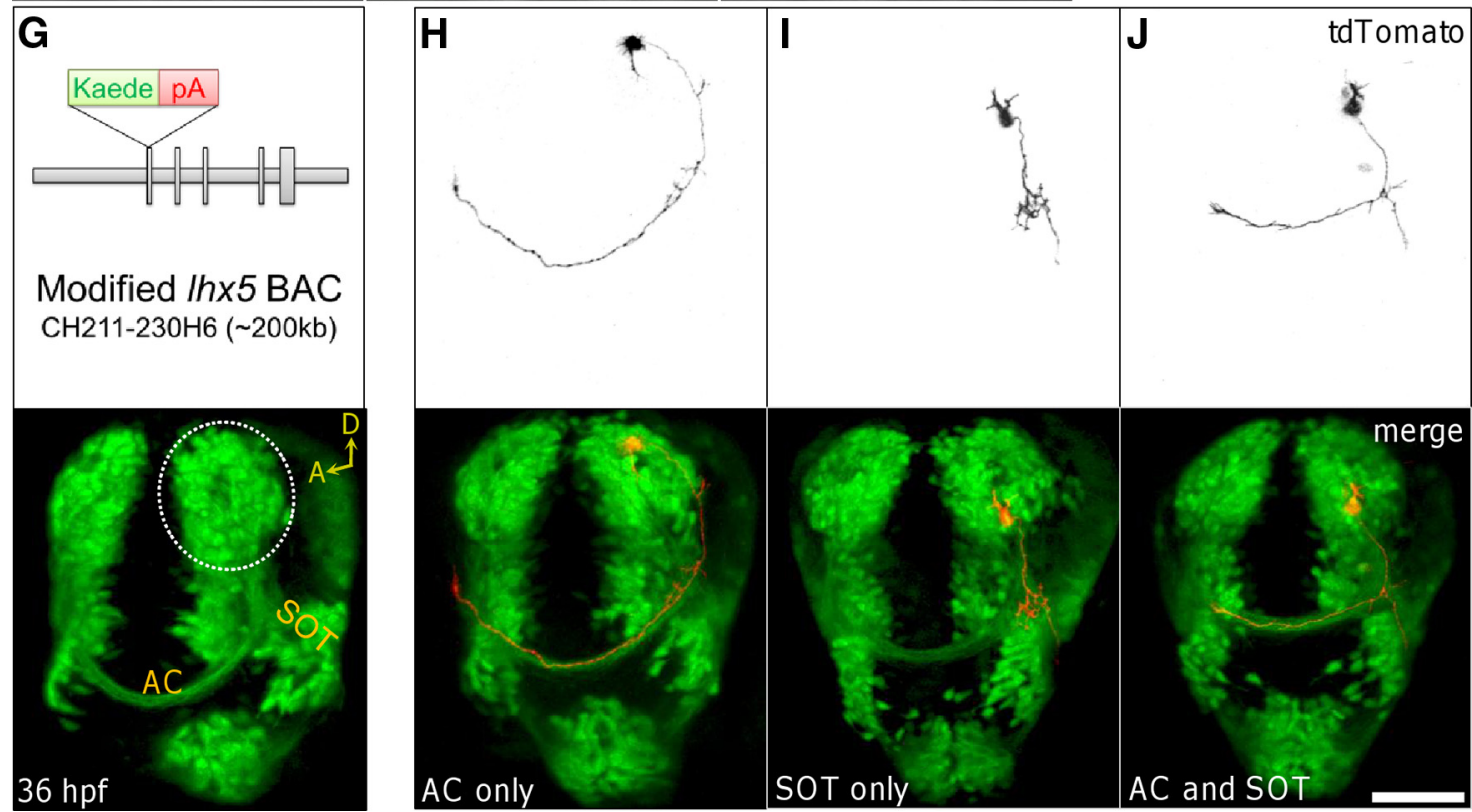

Figure 1. Distinct axonal projection patterns of individually labeled neurons. $\boldsymbol{A}-\boldsymbol{E}$, Heterogeneous axonal projection patterns of forebrain neurons. $\boldsymbol{A}$, Acetylated tubulin antibodies label the $\mathrm{AC}$ and the SOT in a pharyngula period ( $36 \mathrm{hpf}$ ) embryo (frontal view, dorsal is to the top). $\boldsymbol{B}$, Single-cell labeling via mosaic expression of fluorescent protein. Forebrain specific emx3 enhancer drives expression of transcriptional activator Gal4FF in forebrain. Gal4FF binds onto the UAS elements to drive EGFP or tdTomato expression. $C-E$, Individual forebrain neurons labeled by EGFP show different axonal projection patterns. $\boldsymbol{F}$, Schematic view of ADt neurons and their axonal projections along the AC and the SOT. G-J, Distinct axonal projection patterns of individual ADt neurons. $\mathbf{G}$, The Ihx5:Kaede transgenic line labels the neurons located at the anterior dorsal region of the telencephalon. The Kaede expression cassette replaced the first exon of the $/ \mathrm{h} \times 5 \mathrm{gene}$. A live $36 \mathrm{hpf}$ Ihx5:Kaede transgenic embryo was mounted in a tilted frontal view (with a sideways rotation from the frontal view) to reveal the AC and the SOT simultaneously. The white circle marks the left ADt region. D, Dorsal; $A$, anterior. $\boldsymbol{H}-\boldsymbol{J}$, Mosaic expression of tdTomato show three distinct axonal projection patterns of individually labeled ADt neurons. Images of the tdTomato channel are shown in the top panels. Merged images of tdTomato and Kaede channels are shown in the bottom panels. Scale bar: (in $J) A, C-E, G-J, 50 \mu \mathrm{m}$.

\section{Results}

Mosaic labeling of forebrain neurons reveals axonal projection patterns of individual neurons

In the embryonic zebrafish telencephalon, the early born neurons are specified as a cluster of cells in the dorsal rostral region of the neural tube. By the pharyngula period, the telencephalic neurons extended axons into the AC and the SOT (Fig. 1A) (Chitnis and Kuwada, 1990; Wilson et al., 1990). To examine the axonal projection patterns of single neurons, we labeled the forebrain neurons with a transient transgenic method (Fig. $1 B$ ). We coinjected a plasmid carrying emx3:Gal4FF and a plasmid carrying UAS: EGFP into embryos at the one-cell stage. The emx3:Gal4FF plasmid carries a Gal4 transcriptional activator variant (Asakawa et al., 2008) under control of a forebrain-specific enhancer element from the $e m \times 3$ gene (Viktorin et al., 2009). The UAS:EGFP plasmid carries fluorescent protein EGFP under control of five copies of the Gal4 recognition element UAS. Gal4FF acts on the UAS elements to drive EGFP expression in the forebrain. Because plasmids injected into the early stage zebrafish embryos were mosaically distributed among the dividing cells, mosaic expression of fluorescence protein encoded by the injected plasmids may result in random labeling of single or small numbers of cells in the injected embryos (Westerfield et al., 1992; Miyasaka et al., 2009). By screening the injected embryos for axons that can be traced and assigned to single-labeled neurons with confocal microscopy, we found that some of the labeled axons crossed the mid- 

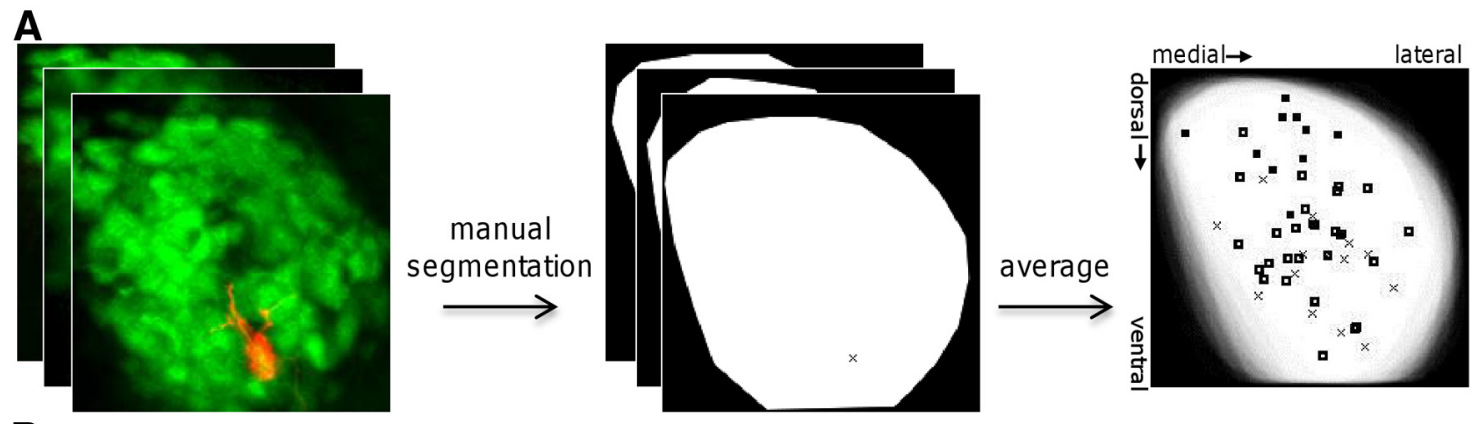

B
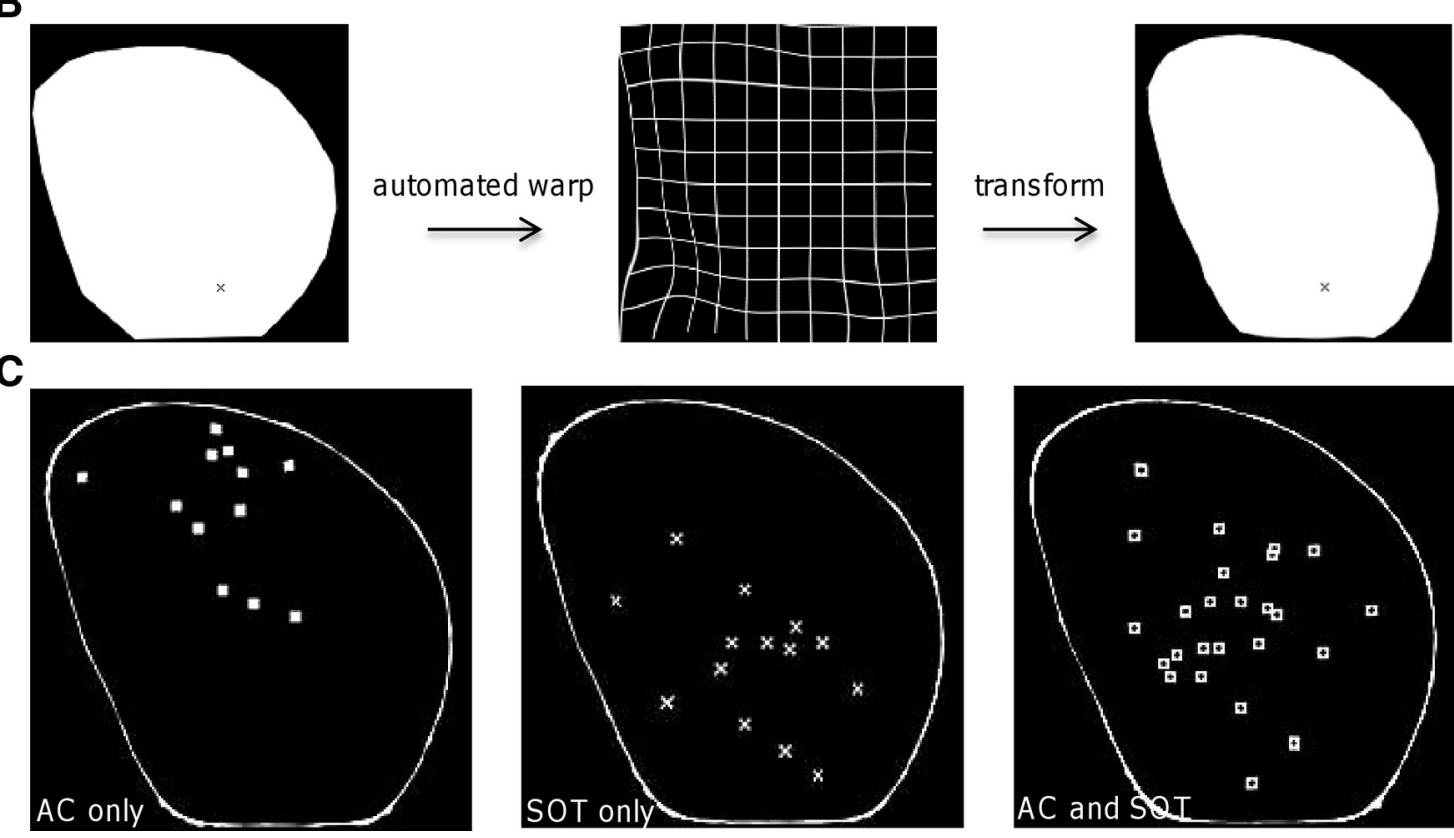

Figure 2. Axonal projection patterns of the ADt neurons correlate with their soma positions in the telencephalon. $A$, Procedures to obtain the common reference image. Images of the ADt regions were manually segmented and then averaged with ImageJ. $\boldsymbol{B}$, Example of image registration. A segmented image was registered to the reference image with a nonrigid method. The middle panel shows the deformation grid for this particular image registration. The nonrigid warping allowed smooth and continuous deformation across the image. C, Distributions of the soma positions of the distinct types of ADt neurons. Soma positions are marked on the common reference image.

line likely along the AC $(n=3$; Fig. $1 C)$, and some axons projected exclusively on the ipsilateral side likely along the SOT $(n=4$; Fig. $1 D)$, whereas other labeled neuronal axons bifurcated and projected into both the AC and the SOT $(n=4$; Fig. $1 E)$.

\section{Axonal projection patterns of the ADt neurons are heterogeneous}

To better examine the heterogeneity of the forebrain neurons, we coinjected the emx3:Gal4FF plasmid and a plasmid carrying UAS: tdTomato into embryos of a transgenic line $\operatorname{Tg}(\operatorname{lh} \times 5 B A C: k a e d e)$ at the one-cell stage (Fig. $1 G-J)$. The $T g(\operatorname{lh} \times 5 B A C$ :kaede) line carries a modified bacteria artificial chromosome (BAC) as a transgene. The modified BAC transgene contains an $\sim 200 \mathrm{~kb}$ sequence surrounding the zebrafish $l h x 5$ gene and in which the first exon of the zebrafish $l h x 5$ gene is replaced by an expression cassette of a fluorescent protein Kaede (referred to as 1 hx5:Kaede hereafter; Fig. 1G) (Gao et al., 2012). The lhx5:Kaede line labels the neurons located at the anterior dorsal region of the telencephalon (Fig. $1 G$, white circle). Thus the Kaede fluorescence labeling provided positional landmarks for localizing the tdTomato-labeled cells. We screened the injected embryos for single-labeled neurons that had somata located in the Kaede fluorescence-marked ADt region and had an axonal projection path clearly labeled. Among these labeled ADt neurons $(N=52), 12$ neurons projected axons into the AC exclusively (Fig. $1 H$ ), 14 projected axons in the ipsilateral SOT exclusively (I), and 26 neurons projected axons into both the AC and the ipsilateral SOT $(J)$. These results suggest that the ADt neurons are likely composed of three distinct populations of projection neurons based on their axonal projection patterns.

\section{Axonal projection patterns of the ADt neurons correlate with} the positions of their somata

To summarize and quantify our results, we sought to bring images of different ADt neuron specimens into a common reference space. We reconstructed each of the 52 confocal image stacks and projected the reconstructed three-dimensional images to a frontal view based on the Kaede fluorescence. We then manually segmented the ADt region in the projected images based on the Kaede labeling and obtained an averaged image of the 52 segmented images (Fig. $2 \mathrm{~A}$ ). The averaged image served as the reference image, and the coordinates along the horizontal and the vertical axes of the reference image represented the standardized medial-lateral and dorsal-ventral positions, respectively (Fig. $2 A)$. We next used an automated nonrigid image registration method (Klein et al., 2011) to align each of the 52 segmented images to the reference image (Fig. $2 \mathrm{~B}$ ). We then obtained the standardized coordinates of the labeled ADt neurons on the com- 
mon reference space (Fig. 2C). Our results showed that the somata of the ADt neurons with exclusive AC-projecting axons were clustered at more dorsal and medial positions (Fig. 2C, AC only), the somata of the ADt neurons with exclusive SOTprojecting axon were clustered at more ventral and lateral positions (Fig. 2C, SOT only), and the ones with both $\mathrm{AC}$ and SOT projections were more widely distributed (Fig. 2C, AC and SOT). ANOVA statistical tests confirmed these differential distributions were significantly different along the dorsal-ventral axis $\left(F_{(2,49)}\right.$ $\left.=18.22 ; p=1.21 \times 10^{-6}\right)$. The distributions along the mediallateral axis, however, were not significantly different $\left(F_{(2,49)}=\right.$ $1.44 ; p=0.248$ ). Post hoc analyses (with Bonferroni's correction) revealed that the somata of the $\mathrm{ADt}$ neurons that projected axons exclusively into the $\mathrm{AC}$ were located at more dorsal positions than those $\mathrm{ADt}$ neurons that projected axons into the SOT only $\left(p=1.89 \times 10^{-6}\right)$ or into both the AC and the SOT $(p=2.27 \times$ $\left.10^{-5}\right)$. Similar statistics results were obtained with soma position data determined from the pretransformed images $\left(F_{(2,49)}=18.46\right.$ $\left.p=1.06 \times 10^{-6}\right)$ (data not shown). The correlation between the axonal projection patterns and the positions of the somata of the ADt neurons suggested that cell intrinsic differences regulate the development of the ADt neurons. These results further indicated intrinsic differences along the dorsal-ventral axis determined the ADt axonal projection choice at the intersection between the AC and the SOT.

\section{Dcc and Robo2 are differentially expressed in the ADt neurons}

To begin to define the molecular identity of the intrinsic differences along the dorsal-ventral axis of the ADt region, we examined expression profiles of two guidance receptors Dcc and Robo2. Both Dcc and Robo2 are expressed in the CNS during zebrafish embryonic development (Lee et al., 2001; Fricke and Chien, 2005). Double labeling by fluorescent in situ hybridization showed that $d c c$ and robo2 were differentially expressed in the dorsal telencephalon (Fig. 3). At the beginning of the pharyngula period, when the ADt neuronal axons started to extend ventrally (24hpf), $d c c$ transcripts were more enriched in the dorsal and medial regions in the dorsal telencephalon. In contrast, robo 2 transcripts were located in more ventral and lateral positions to the $d c c$ transcripts (Fig. 3A-C). At $36 \mathrm{hpf}$ after the ADt neuronal axons extended into the AC and the SOT pathway, $d c c$ and robo2 were similarly differentially expressed in dorsal telencephalon (Fig. 3D-F). Together with our cell-labeling results above, these expression patterns suggested that the ADt neurons with exclusively AC-projecting axons likely expressed $\mathrm{Dcc}$, while those $\mathrm{ADt}$ neurons with SOT-projecting axons likely expressed Robo2.

To further examine whether the ADt axon projections correlated with Dcc and Robo2 expression, we performed single-celllabeling experiments and determined the Dcc and Robo2 expression with in situ hybridizations. Because the tdTomato fluorescence did not survive the in situ hybridization procedure, the mosaically labeled single cells and axonal projections were stained with an anti-DsRed antibody, which recognized the tdTomato protein (Fig. $3 H-P$ ). Triple-labeling results showed that ADt axon projections correlated with Dcc and Robo2 expression profiles [Fig. 3Q; $\chi_{(4)}^{2}=40.49, n=43 ; p=5.06 \times 10^{-9}$ ]. Pairwise Fisher's exact tests showed the Dcc and Robo 2 expression profiles were significantly different between the three types of ADt neurons $\left(\mathrm{AC}\right.$ vs $\mathrm{AC}-\mathrm{SOT}, p=6.35 \times 10^{-4}$; AC vs SOT, $p=1.68 \times$ $10^{-5}$; AC-SOT vs SOT, $\left.p=4.19 \times 10^{-4}\right)$. Thus, $60 \%$ of the labeled ADt neurons with AC-projecting axons expressed $d c c$ selectively ( 6 of 10; Fig. $3 H-J$ ), and none expressed robo2 exclu- sively. In contrast, most (83\%) of the labeled ADt neurons with both AC- and SOT-projecting axons expressed both $d c c$ and robo2 (15 of 18; Fig. $3 K-M)$, and most (80\%) of the labeled ADt neurons with SOT-projecting axons expressed robo2 selectively $(12$ of 15 ; Fig. $3 N-P$ ). In addition, none of the AC-SOT- or the SOT-type ADt neurons expressed $d c c$ exclusively.

\section{Knocking down Dcc function does not prevent formation of the $\mathrm{AC}$ and the SOT originating from the ADt neurons}

We next examined whether inhibition of Dcc or Robo2 function may affect the axonal projection patterns of the ADt neurons. To facilitate our investigation, we took advantage of the lhx5:Kaede transgenic line and the photoconvertible property of the Kaede fluorescence protein (Ando et al., 2002). We photoconverted Kaede protein in the ADt neuronal somata from the green light emission form to the red light emission form. Diffusion of the photoconverted, red-colored Kaede from the ADt somata into the axons marked the AC and the SOT axonal tracts that originated from the ADt neurons (Fig. 4A,B).

To inhibit Dcc function, we injected a translation-blocking morpholino antisense oligonucleotides [described by Suli et al. (2006); referred to as $d c c$-MO1 hereafter] into zebrafish embryos at one- to four-cell stages. Photoconversion of Kaede in dccMO1-injected lhx5:Kaede animals showed that both the AC and the SOT were formed $(n=60 ; \mathrm{Fig} .4 D)$. In addition to the $\mathrm{AC}$ and the SOT, an aberrant axon tract was also observed in the injected embryos (Fig. $4 D$, arrow).

We examined the effectiveness of the $d c c$ morpholino knockdown with a rabbit anti-zebrafish Dcc antibody. By Western blotting, this antibody detected the endogenous Dcc protein as a band of $\sim 170 \mathrm{~kb}$ in the whole embryo protein extract, and the specificity of the antibody was corroborated by detection of the Myc-tagged and GFP-tagged Dcc protein (data not shown). In embryos injected with the $d c c$-MO1, the expression of the Dcc was reduced to levels below the detection limits of the Western blots (Fig. $4 D$, inset). This antibody did not work on wholemount embryos or frozen sections of embryos.

We further examined the ADt neuronal axons with singlecell-labeling when Dcc expression was knocked down with the $d c c-\mathrm{MO} 1$ injection. Among the labeled ADt neurons in $d c c-\mathrm{MO}-$ injected embryos ( $n=50), \sim 48 \%$ of the labeled neurons either failed to extend axons ventrally or had axons stalled at the junction between the AC and the SOT (Fig. $4 E-H$ ). The rest of the labeled ADt neurons extended axons into the $\mathrm{AC}$ and the SOT in patterns similar to those of the wild-type controls (Fig. 4I,J). The somata of the ADt neurons with exclusive AC- or SOT-projecting axons were clustered at more dorsal (Fig. 4I, AC only) or at more ventral (Fig. 4I, SOT only) positions, respectively, and the ones with both $\mathrm{AC}$ and SOT projections were more widely distributed (Fig. 4J, AC and SOT). ANOVA and Student's $t$ test results showed that these distribution patterns were not significant different from those of wild-type controls (ANOVA test along the dorsal-ventral axis, $F_{(2,24)}=4.861, p=0.017$; along medial-lateral axis, $F_{(2,24)}=1.354, p=0.277 ; t$ test for the $\mathrm{AC}$ only type, $p=0.106$; SOT only type, $p=0.439$; $\mathrm{AC}$ and the SOT type, $p=0.073$ )

To address the specificity of the phenotypes caused by the morpholino knockdown of Dcc, we tested for rescue of the aberrant axon tract by coinjection of a dcc-MO1-resistant mRNA containing silent mutations in the morpholino target site (Fig. $4 K)$. After coinjection of the $d c c$-MO1 and the morpholinoresistant $d c c$ mRNA, the percentage of the embryos with aberrant axon tracts was significantly reduced (from $64 \pm 12 \%, n=60$ to 

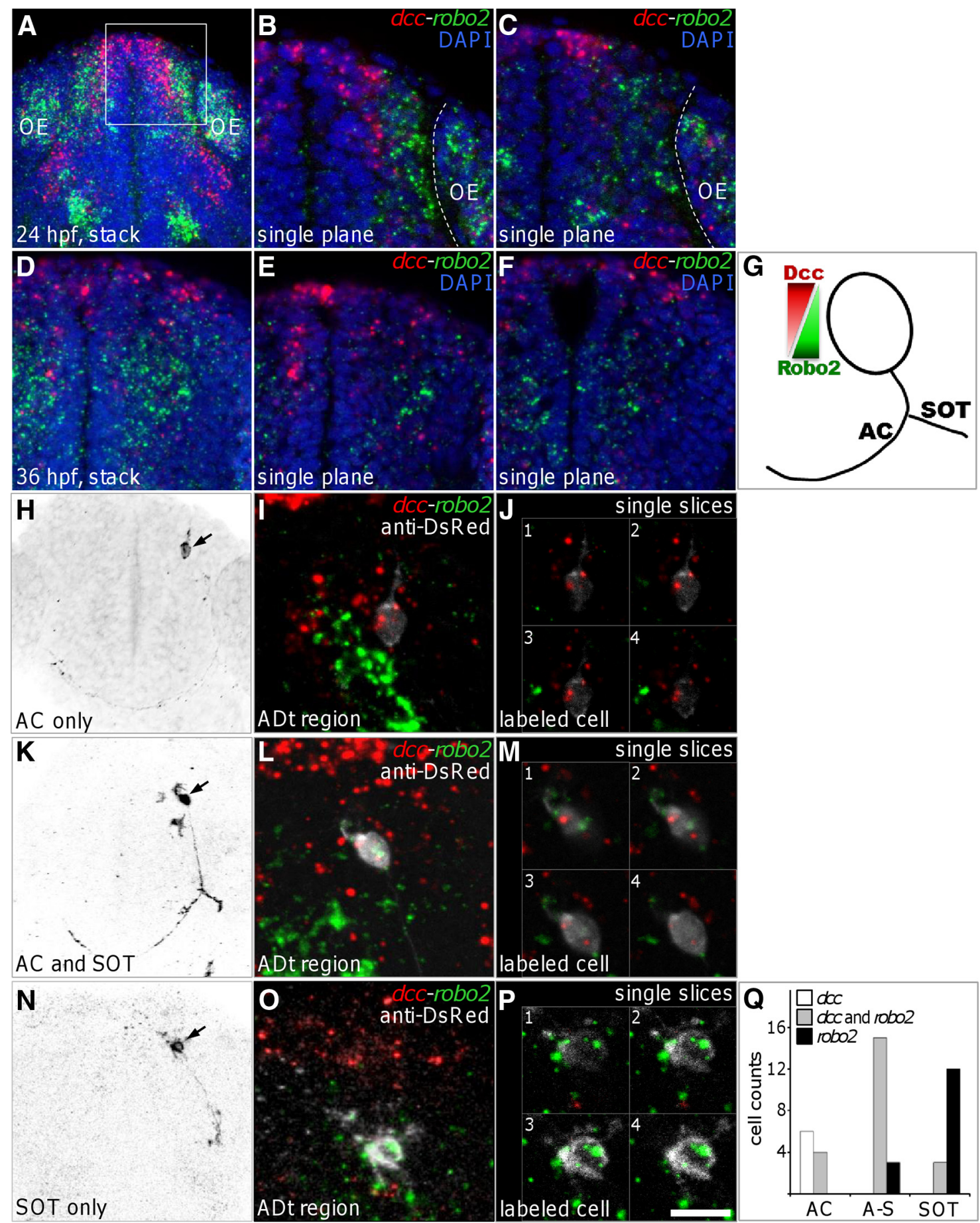

Figure 3. Dcc and Robo2 are differentially expressed in the dorsal telencephalon. $A-C$, Dcc and Robo2 expression patterns in the telencephalon at 24 hpf. The embryo was counterstained with DAPI to show cell nuclei (frontal view, dorsal to the top). The dorsal telencephalon region in $\boldsymbol{A}$ is marked with a white square, and the details within the square are shown in $\boldsymbol{B}$ and $\boldsymbol{C}$. A projection of the image stack is shown in $\boldsymbol{A}$; images of single confocal planes are shown in $\boldsymbol{B}$ and $\boldsymbol{C}$. The confocal plane of $\boldsymbol{B}$ is anterior to that of $\boldsymbol{C}$. White dash lines in $\boldsymbol{B}$ and $\boldsymbol{C}$ demarcate the border between the telencephalon and the olfactory epithelium (OE). D-F, Dcc and Rob02 expression patterns in the telencephalon at $36 \mathrm{hpf}$ (frontal view, dorsal to the top). A projection of the image stack is shown in $\boldsymbol{D}$; images of single confocal planes are shown in $\boldsymbol{E}$ and $\boldsymbol{F}$. The confocal plane of $\boldsymbol{E}$ is anterior to the $\boldsymbol{F}$ plane. $\boldsymbol{G}$, Schematic view of $\mathrm{Dcc}$ and Robo2 expression patterns in the $A D t$ neurons. Transcripts for dcc are enriched in the dorsal and medial regions, and robo2 transcripts are distributed more in the ventral and lateral positions in the dorsal telencephalon. $\boldsymbol{H}-\mathbf{Q}$, Correlations of ADt axonal projections and Dcc and Robo2 expression profiles. Single-cell-labeled embryos were triple stained with dcc/robo2 in situ probes and anti-DsRed antibodies. The anti-DsRed show labeled neurons and their axon projections $(\boldsymbol{H}, \boldsymbol{K}, \boldsymbol{N})$. Arrow marks the cell body position of the labeled neuron. Corresponding merged panels $(\boldsymbol{I}, \boldsymbol{L}, \boldsymbol{O})$ show projected images of confocal image stacks of the triple labeling in the ADt regions. Labeled cell panels $(J, M, P)$ show four $(1-4)$ consecutive single slices of confocal image stacks that go through the labeled cell bodies. $\boldsymbol{Q}$, Labeled cells are counted as expressing Dcc selectively (open bar), expressing both Dcc and Robo2 (gray bar), or expressing Robo2 selectively (black bar). Every single slice of confocal image stacks was inspected for presence of Dcc and Robo2 signals within the anti-DsRed-labeled cell bodies. A-S, AC and SOT. See Results for detailed statistical test results. Scale bar: (in $\boldsymbol{P}) \boldsymbol{A}, \boldsymbol{H}, \boldsymbol{K}, \boldsymbol{N}, 50 \mu \mathrm{m} ; \boldsymbol{B}, \mathbf{C}, \boldsymbol{I}, \mathbf{L}, \mathbf{O}, 20 \mu \mathrm{m} ;$ in $\mathbf{D}-\boldsymbol{F}, 25 \mu \mathrm{m} ; \boldsymbol{J}$, $M, P, 12 \mu \mathrm{m}$. 

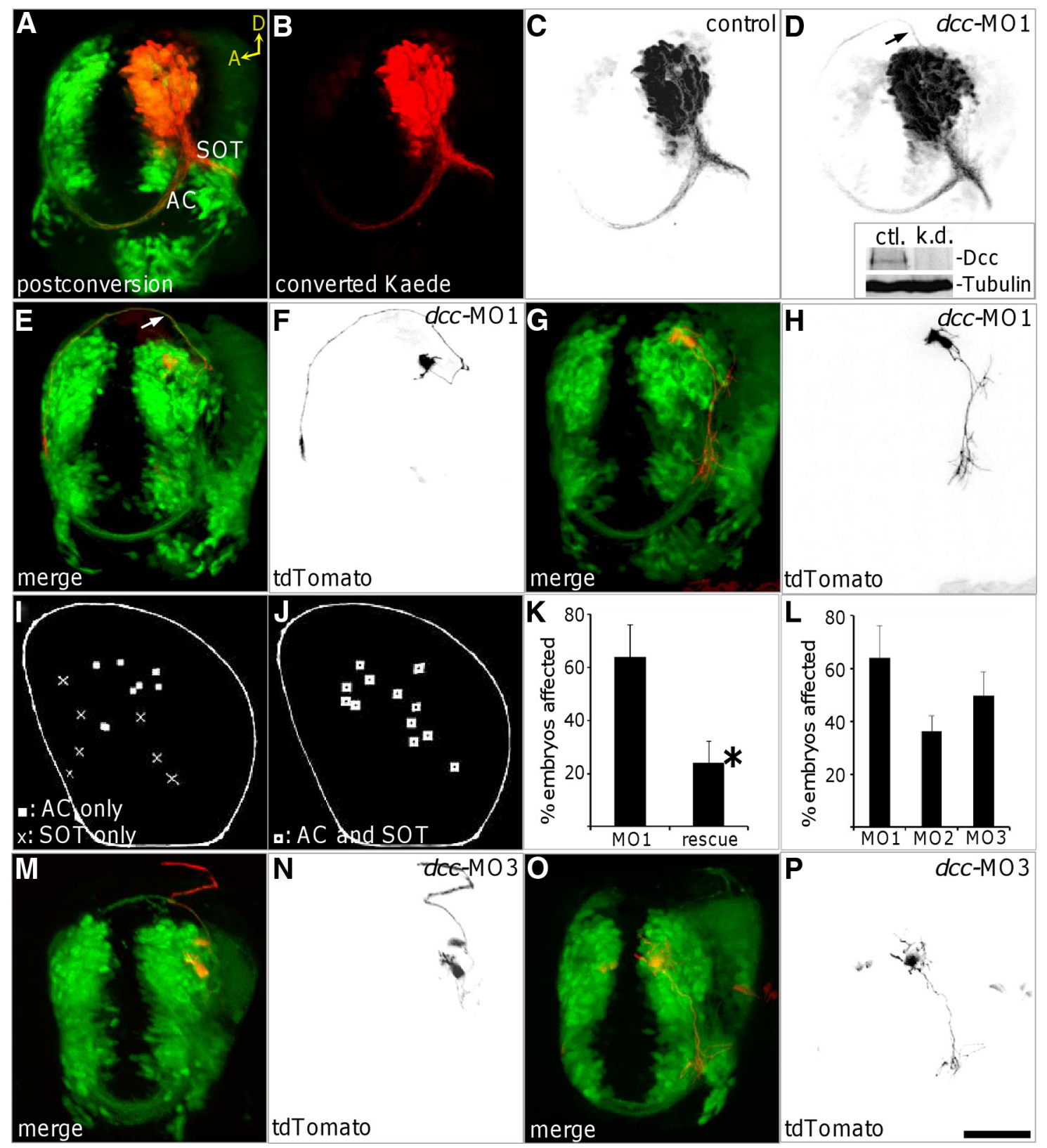

Figure 4. $\mathrm{AC}$ and SOT are present in dcc-MO-injected embryos. $A, B$, Photoconversion of Kaede in an Ihx5:Kaede transgenic embryo labels the AC and the SOT originating from the ADt neurons. A live $36 \mathrm{hpf} / \mathrm{h} \times 5$ :Kaede transgenic embryo was mounted in the tilted frontal view, and the region of the left telencephalon was selected for photoconversion. $C, D$, Knocking down Dcc function does not prevent the formation of the AC and the SOT. The arrow in $\boldsymbol{D}$ marks an aberrant axon tract after knockdown of Dcc function. The inset in $\boldsymbol{D}$ shows expression of the Dcc protein was effectively reduced by the morpholino knockdown (k.d.). ctl, Uninjected control embryos. Tubulin labels served as a loading control. $E, F$, ADt neuronal axon fails to reach the ventral positions in a dcc-M01-injected embryo. G, $\boldsymbol{H}$, ADt neuronal axon stalls at the junction between the AC and the SOT in a dcc-M01-injected embryo. $I, J$, Distributions of the soma positions of the distinct types of ADt neurons in embryos injected with dcc-M01. The distribution patterns are similar to those of the standard controls. See Results section for statistic test results. $K$, Rescue of $d c c-M 01$ knockdown phenotype by a morpholino-resistant dcc mRNA. ${ }^{*} p<0.05$. M01, dcc-M01 injected; rescue, dcc-M01 and morpholino-resistant dcc mRNA coinjected. L, dcc-M02 or dcc-M03 causes similar phenotypes as dcc-M01 injection. $\boldsymbol{M}, \boldsymbol{N}, \mathrm{ADt}$ neuronal axon extends dorsally in a dcc-M03-injected embryo. $\mathbf{0}, \boldsymbol{P}, \mathrm{ADt}$ neuronal axon stalls at the junction between the AC and the SOT in a dcc-M03-injected embryo. Scale bar: (in $\boldsymbol{P}) \boldsymbol{A}-\boldsymbol{D}, \boldsymbol{M}-\boldsymbol{P}, 50 \mu \mathrm{m} ; \boldsymbol{E}-\boldsymbol{H}, 42 \mu \mathrm{m}$.

$24 \pm 8 \%, n=41 ; p=0.005)$. To further examine the specificity of the morpholino knockdowns, we tested a second nonoverlapping translation-blocking morpholino $(d c c-\mathrm{MO} 2)$ and a spliceblocking morpholino ( $d c c-\mathrm{MO} 3)$ that have been described previously (Suli et al., 2006). Similar to dcc-MO1-injected embryos, aberrant axon tracts were observed in the dorsal telencephalon in $d c c-\mathrm{MO} 2-$ or $d c c$-MO3-injected embryos $(36 \pm 6 \%, n=64$ or $50 \pm 9 \% n=68$, respectively; Fig. $4 L$ ). We also performed singlecell-labeling in $d c c-\mathrm{MO} 2$ - or $d c c-\mathrm{MO} 3$-injected embryos. Similar to $d c c$-MO1-injected embryos, $\sim 24 \%$ of the labeled neurons in $d c c-\mathrm{MO} 2$-injected embryos (5 of 21 ) or $41 \%$ of the labeled neurons in dcc-MO3-injected embryos (16 of 39) either failed to extend axons ventrally or had stalled axons (Fig. $4 M-P$ ).

\section{Knocking down Robo2 function abolishes the SOT} originating from the ADt neurons

To inhibit Robo2 function, we injected a morpholino that targets the translation start site of the robo2 mRNA (robo2-tMO) into the lhx5:Kaede embryos (Fig. 5A-C). Photoconversion of Kaede in robo2-tMO-injected embryos $(n=36)$ showed that the SOTs 

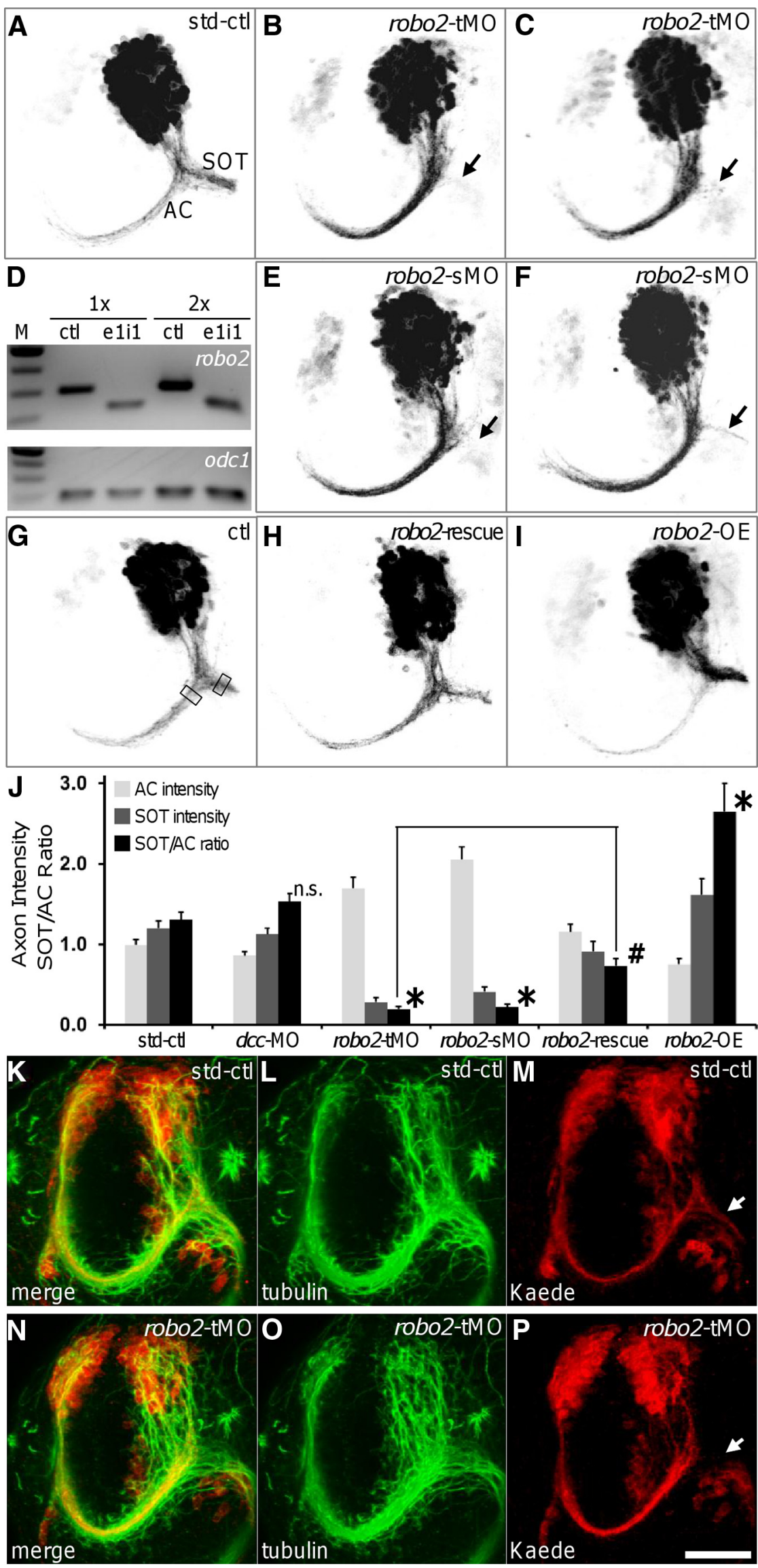

Figure 5. Knocking down Rob02 abolishes formation of SOT originating from the ADt neurons. $A$, Photoconverted Kaede labels the $\mathrm{AC}$ and the SOT in a standard control morpholino-injected embryo (std-ctl). $\boldsymbol{B}, \boldsymbol{C}$, The SOT is lost $(\boldsymbol{B})$ or severely reduced $(\boldsymbol{C})$ in were significantly affected in most of the injected embryos (97\%); the SOTs were either absent (61\%; Fig. $5 B)$ or greatly reduced (36\%; Fig. 5C). To validate the specificity of the robo2-tMO knockdowns, we examined phenotypes caused by injection of a standard control morpholino and a splice-blocking morpholino against the first exon-intron junction of the robo2 transcripts (robo2-sMO). None of the embryos injected with the standard control morpholino showed defects in the SOT or the AC ( $n=55$; Fig. $5 A)$. In embryos injected with the splice-blocking robo2sMO $(n=34)$, the SOTs were either severely reduced $(50 \%$, Fig. $5 F)$ or absent (44\%, $n=36$; Fig. 5E). RT-PCR analysis showed that the wild-type robo2 transcripts were lost and replaced by shorter robo2 transcripts in the robo2-sMOinjected embryos (Fig. 5D). Sequencing analysis indicated that the shorter robo2 transcripts missed $81 \mathrm{bp}$ and were due to usage of a cryptic splice donor site. Because the aberrantly spliced RNA lacked the wild-type start codon, it was not expected to encode functional proteins (data not shown). A previous study using a different morpholino against the first

\section{$\leftarrow$}

robo2-tM0-injected embryos. Arrows point to positions of expected SOTs. Live embryos were mounted as in Figure 4A. $\boldsymbol{D}$, RT-PCR results show an aberrant splice of robo2 transcripts in the splice-blocking robo2-sM0-injected embryos. The aberrantly spliced robo2 transcripts are smaller and lack the start codon in the wild-type transcripts. The amplification of odc 1 gene served as a loading control. Lanes marked $2 \times$ are from reactions using twice the amount of CDNA template as lanes marked by $1 \times$. ctl, Control; $\mathrm{M}$, size marker; e1i1, robo2-sM0 injected. $\boldsymbol{E}, \boldsymbol{F}$, The SOT is lost $(\boldsymbol{E})$ or severely reduced $(\boldsymbol{F})$ in the splice-blocking robo2-sM0-injected embryos. Arrows point to positions of expected SOTs. Live embryos were mounted as in Figure 4A. G, A control embryo injected with water. Boxes indicate the selected $\mathrm{AC}$ and $\mathrm{SOT}$ regions for quantification. $\boldsymbol{H}$, Coinjection of a robo2 morpholino-resistant mRNA (robo2rescue) restores the SOT formation in embryos injected with the robo2-tM0. $I$, Robo2 overexpression (robo2-0E) causes reduction of the AC and enhancement of the SOT. J, Quantification of the AC, SOT and SOT/AC ratios. Axon intensity values are presented in arbitrary units. Error bars indicate SEs. Numbers of animals analyzed for each treatment group are $n=42,60$, $36,34,30$, and 30 , respectively. ${ }^{*} p<0.05$ versus standard controls by Student's $t$ test. n.s., Not significant, $p>0.05$ versus standard controls. $" p<0.05$ between the robo2-tM0 and the robo2-rescue group (and bracket). Detailed statistic test results are reported in Results. $\boldsymbol{K}-\boldsymbol{M}$, Double staining of acetylated tubulin and Kaede in a standard control morpholino-injected embryo. The acetylated tubulin-labeled axon tracts are broader than those labeled by Kaede antibodies. The arrow in $M$ points to the SOT labeled by the Kaede antibodies. $\boldsymbol{N}-\boldsymbol{P}$, Double staining of acetylated tubulin and Kaede in a robo2-tMO-injected embryo. The acetylated tubulin-labeled SOT axons are present $(\mathbf{0})$. The Kaedelabeled axons are absent at the expected position (arrow) for the SOT (P). Scale bar: (in $\boldsymbol{P}) \boldsymbol{K}-\boldsymbol{P}, 50 \mu \mathrm{m}$. 

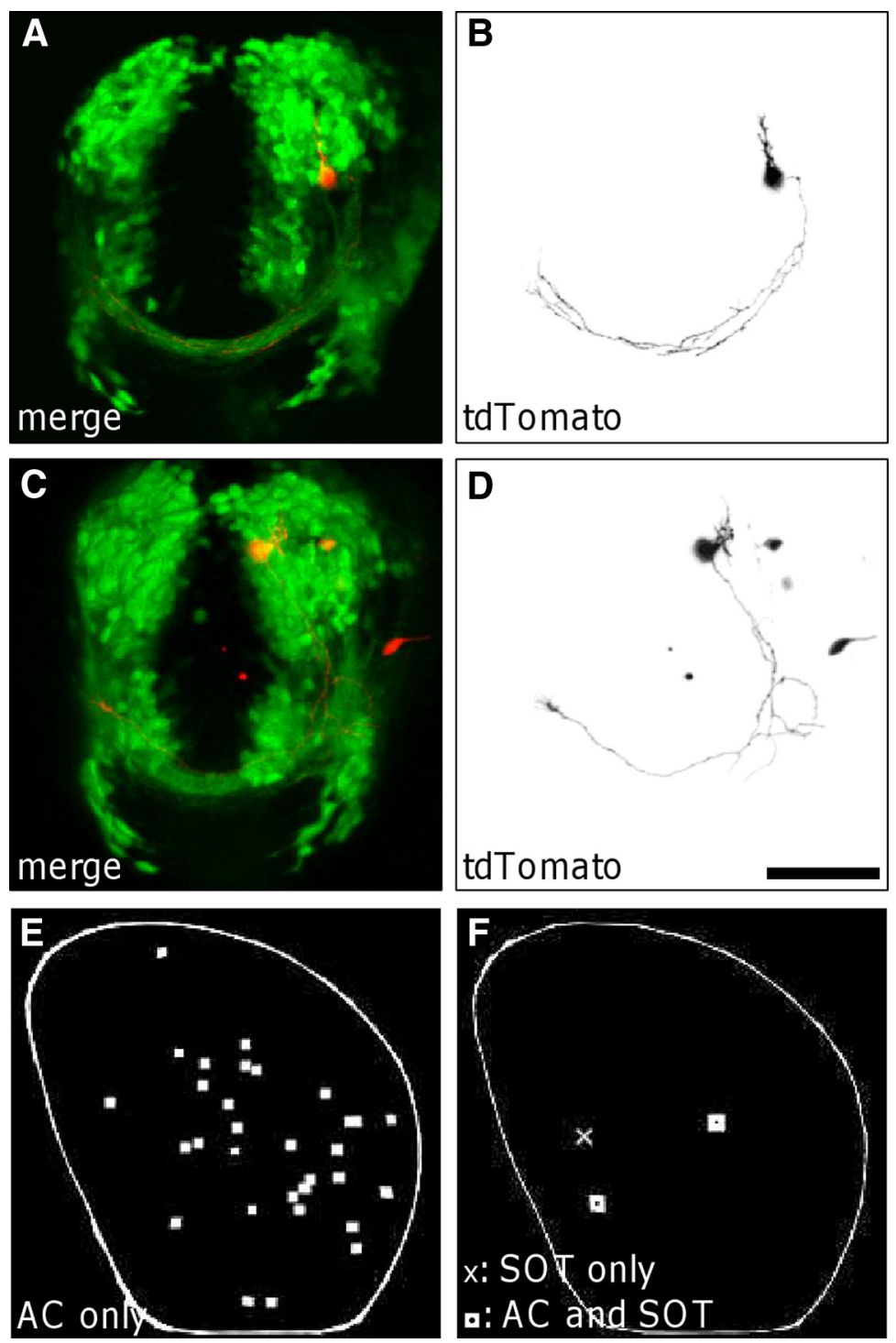

Figure 6. Individually labeled ADt neurons project axons into the AC when Robo2 function is inhibited. $\boldsymbol{A}, \boldsymbol{B}, \mathrm{A}$ single-celllabeled ADt neuronal axon bifurcates at the $A C-S O T$ junction, and both branches project into the $A C$ in a robo2-tM0-injected embryo. $\boldsymbol{C}, \boldsymbol{D}, A$ single-cell-labeled $A D$ t neuron with one axonal branch projects into the $A C$, and the other branch wanders at the $\mathrm{AC}-\mathrm{SOT}$ junction in a robo2-tM0-injected embryo. Scale bar: (in $\boldsymbol{D}) \boldsymbol{A}-\boldsymbol{D}, 50 \mu \mathrm{m}$. $\boldsymbol{E}, \boldsymbol{F}$, Distribution of soma positions of distinct types of ADt neurons in robo2-tM0-injected embryos. Most labeled ADt neurons project axons exclusively into the AC. compared with the controls (Fig. 5J), the robo2 morpholino knockdowns caused an approximately threefold to fourfold reduction in the SOT intensity, an $\sim 75-$ $100 \%$ increase of the AC tract intensity, and an approximately sevenfold reduction in the mean value of the SOT/AC ratios $\left(p=1.64 \times 10^{-15}\right.$ and $p=3.68 \times$ $\left.10^{-15}\right)$. Overexpression of Robo2 by mRNA injection caused an $\sim 40 \%$ increase of the SOT intensity, 25\% decrease of the AC tract intensity, and a more than twofold increase of the mean value of the SOT/AC ratios $(p=0.001)$. The mean value of the SOT/AC ratios of the robo2rescue group increased approximately fourfold compared to the group injected with the robo2-tMO alone $\left(p=1.76 \times 10^{-6}\right)$, and the SOT and the AC tract intensities were restored to levels comparable to those in the control embryos $(p=0.823$ and $p=$ 0.129 , respectively).

A previous study showed that the SOT labeled by the acetylated tubulin appeared unaffected after the function of Robo 2 was inhibited by morpholino injections (Devine and Key, 2008). We thus fixed the standard control morpholino or robo2$\mathrm{MO}$-injected embryos and costained the fixed embryos with acetylated tubulin and Kaede antibodies. Our results showed that in the robo2-tMO-injected embryos, the acetylated tubulin-labeled axonal tracts were present between the telencephalon and the diencephalon (Fig. 5L,O). In contrast, the Kaede-labeled axonal tracts were missing or dramatically reduced (Fig. $5 M, P$ ). Similar results were obtained with the injection of the spliceblocking robo2-sMO (data not shown). Therefore, our results showed that Robo2 function was specifically required for the ADt neurons to extend the axons along the SOT pathway. exon-intron junction of the robo2 gene showed the same effects on the splice of robo2 transcripts (Wyatt et al., 2010). These results confirmed that the high penetrance $(>90 \%)$ loss or severe reduction of the SOT in robo2-MO-injected embryos was due to specific knockdowns of Robo2 function.

To further address the specificity of the morpholino knockdowns, we tested for rescue of the robo 2 knockdown phenotypes by coinjection of a robo2-tMO-resistant mRNA harboring seven silent mutations in the morpholino recognition site (Fig. $5 \mathrm{H}$, robo2-rescue). After coinjection of the robo2-tMO and the morpholino-resistant robo $2 \mathrm{mRNA}$, the SOT was restored in $\sim 67 \%$ of the injected embryos $(n=30)$. Interestingly, overexpression of Robo 2 by injection of robo 2 mRNA alone caused reduction of the $\mathrm{AC}$ and enhancement of the SOT (Fig. 5I). To quantify these loss-of-function and gain-of-function phenotypes above, we quantified the intensity of the SOT and the AC tracts, and the intensity ratios between the SOT and the AC tract in each embryo (Fig. 5G, boxes) (see Materials and Methods). Thus,
ADt neurons project axons into the $\mathrm{AC}$ when Robo2 function is inhibited

We examined the axonal projection patterns of singly labeled ADt neurons when the Robo2 function was knocked down (Fig. 6). A majority of the singly labeled ADt neurons (91\%, $n=34$ ) projected axons exclusively into the AC, whereas very few labeled ADt neuronal axons projected exclusively into the SOT $(3 \%)$ or bifurcated into both the AC and the SOT (6\%). Interestingly, some of the labeled ADt neurons with exclusively AC-projecting axons had multiple branches at the intersection between the AC and the SOT $(n=10)$. Those multiple processes either projected into the $\mathrm{AC}$ (Fig. $6 A, B$ ), or the branches wandered around the intersection but failed to enter the SOT (Fig. 6C,D).

We next determined the soma positions of those labeled ADt neurons. In contrast to results in wild-type animals (Fig. $2 C$ ), the somata of the ADt neurons with exclusive AC-projecting axons in robo2-tMO-injected embryos were no longer clustered at the dorsal positions and were broadly distributed instead (Fig. 6E). 
ANOVA tests confirmed that the somata positions of the ADt neurons with exclusive AC-projecting axons were significantly different between the wild-type and the robo2-MO-injected em$\operatorname{bryos}\left(F_{(1,40)}=23.00 ; p=2.26 \times 10^{-5}\right)$. Additional ANOVAs showed that the distribution of the AC-projecting ADt neurons in robo2-MO-injected embryos were similar to those of the SOTprojecting or AC- and SOT-projecting ADt neurons in the wild-type animals $\left(F_{(2,67)}=1.06 ; p=0.352\right)$. Thus these single-cell-labeling results mirrored the group-labeling results with Kaede conversion. Together, these results suggested that the $\mathrm{ADt}$ axons that should extend along the SOT projected into the AC instead when the Robo2 function was inhibited.

\section{Inhibition of Slit or Netrin function affects the SOT or AC formation, respectively}

The Robo receptor ligands Slits are chemorepellents that prevent the axons from extending toward the midline (Bagri et al., 2002). We investigated whether inhibition of Slit function similarly affected the SOT formation as did the inhibition of Robo2 function. There are four Slit homologs in zebrafish, and they are differentially expressed in the CNS during zebrafish embryonic development (Yeo et al., 2001; Hutson et al., 2003). We examined the expression of these four Slit homologs in the forebrain regions with whole-mount in situ hybridizations. The results showed that Slitla was expressed in both the dorsal and ventral telencephalon in the early pharyngula period ( $24 \mathrm{hpf})$, Slit1b was expressed in a restricted region between the telencephalon and the diencephalon at $36 \mathrm{hpf}$. Morpholino knockdown of Slitla or Slit1b function did not affect the SOT formation. Similar to results from a previous report (Barresi et al., 2005), the AC was variably reduced in some Slit1a morpholino-injected embryos (data not shown).

We focused our study on Slit2 and Slit3. Transcripts of Slit2 and Slit3 were enriched in the ventral telencephalons and close to the midline in the early pharyngula period (Fig. $7 A, B$, arrows) (data not shown) (see also Hutson and Chien, 2002). Inhibition of Slit2 function by injecting the slit2 morpholino (Barresi et al., 2005) caused a twofold reduction in the SOT formation (Fig. 7E) and a twofold reduction in the mean value of the SOT/AC ratios (Fig. 7I; $p=1.38 \times 10^{-4}$ vs standard control). Inhibition of Slit3 function alone by injecting the slit3 morpholino (Barresi et al., 2005) did not affect the SOT formation or the SOT/AC ratio (Fig. $7 I ; p=0.64$ and $p=0.10$, respectively). Coinjection of both the slit2 and the slit3 morpholinos caused a further reduction in the mean value of the SOT/AC ratios (Fig. $7 F ; p=3.65 \times 10^{-7}$ vs standard control). The penetrance and severity of the SOT defects caused by the Slit morpholino knockdowns were lower than those in the Robo2 morpholino knockdowns (a penetrance of $68 \%$ in slit2 and slit 3 double knockdowns vs $97 \%$ in robo2-tMO knockdown, and a threefold reduction of the SOT/AC ratio in slit2 and slit3 double knockdowns vs a sevenfold reduction in robo2-tMO knockdown; Fig. 7I). Increasing the injection dosages for Slit morpholinos caused morphological alterations of the embryos and significant reduction of the Kaede fluorescence signal, which impeded the analysis of the ADt axon phenotypes (data not shown).

We next examined whether knocking down the function of the Dcc receptor ligand Netrin affected the AC or the SOT formation. There are four Netrin homologs in zebrafish (Lauderdale et al., 1997; Strähle et al., 1997; Park et al., 2005). At 24 hpf, netrin $1 a$ and netrin $1 b$ are expressed in the ventral and midline regions of the forebrain (Fig. 7C,D) (Lakhina et al., 2012). The other two Netrins, Netrin2 and Netrin4, are not expressed in the forebrain regions before $36 \mathrm{hpf}$ (Park et al., 2005). Inhibition of either Netrin1a or Netrin 1b function by injection of the netrin $1 a$ or the netrin $1 b$ morpholinos (Suli et al., 2006) caused small reductions of the AC axon tract (20 or 25\%) and almost no changes of the SOT axon tract (3\% increase or $8 \%$ decrease, respectively). The mean value of the SOT/AC ratios was increased 30 or $17 \%$ compared to standard controls. Inhibition of Netrin 1 function by coinjecting the netrin $1 a$ and the netrin $1 b$ morpholinos together caused a larger reduction of the $\mathrm{AC}(35 \%)$ and an increase of the SOT $(30 \%)$, and the mean value of the SOT/AC ratios was increased threefold compared to standard controls (Fig. 7G; $p=$ 0.008). Similar to Dcc knockdowns, inhibition of the Netrin1 function also caused the ADt neurons to extend aberrant axons in the dorsal telencephalon (Fig. 7G, arrow).

Our results showed that inhibition of the Robo2-Slit2/Slit3 pathway caused reduction in the SOT formation, while inhibition of the Netrin 1 function caused reduction in the AC tract. To test how the Robo2-Slit2/Slit3 pathway interacted with the Netrin1, we inhibited both Robo2 and Netrin1 function with morpholino injections. The results showed that the SOT was missing or severely reduced. In contrast, the $\mathrm{AC}$ was formed in these embryos (Fig. $7 \mathrm{H}$ ). Quantification results showed the mean SOT intensity was reduced approximately fourfold, while the AC was increased $\sim 60 \%\left(p=1.50 \times 10^{-9}\right.$ and $p=0.007$ vs standard control, respectively). The mean value of the SOT/AC ratios of the robo2/ netrin1 double knockdown embryos was reduced to a level comparable to that of the robo2 single knockdown embryos (Fig. 7I; $p=0.815$ ). Thus, the robo 2 morpholino knockdown was epistatic to the netrin 1 morpholino knockdown in terms of the SOT/AC projection pathway choices of the $\mathrm{ADt}$ neurons. In the robo2/ netrin 1 double knockdown embryos, the ADt neurons also project aberrant axons into the dorsal telencephalon regions (Fig. $7 \mathrm{H}$, arrow).

Finally, to examine the effects of morpholino knockdowns on the specification of the ADt neurons, we determined the expression patterns of $d c c$ and robo2 mRNA in the ADt region in morpholino-injected embryos. We found the $d c c$ transcripts were enriched in the dorsal region, and the robo 2 transcripts were enriched in the ventral region in morpholino-injected embryos (dcc-MO, $n=8$; robo2-tMO, $n=8$; netrin $1 a, b-\mathrm{MO}, n=5$; slit2,3$\mathrm{MO}, n=5$ ). Thus, the expression patterns of $d c c$ and robo2 were similar to those in the wild-type control embryos (Fig. 7J--M). Together with our single-cell-labeling and Kaede conversion results, these results suggested that the incorrect axonal projections observed in our morpholino knockdown experiments were not likely due to abnormal specification of the ADt neurons, because the neurons differentiated along their normal time course and expressed regional markers at appropriate locations.

\section{Discussion}

The results of the present study indicate that intrinsic differences along the dorsal-ventral axis of the telencephalon regulate $\mathrm{ADt}$ neuron axonal pathfinding. Further mechanistic studies suggest that Robo2 responds to the repellent Slit signals and suppresses the attractive Netrin 1 signals, allowing the ADt neurons to extend axons along the SOT pathway (Fig. $8 \mathrm{~A}$ ). Our studies also reveal that in the absence of the attractive Netrin, the ADt neurons can extend axons into the $\mathrm{AC}$ pathway if the repellent Robo2 is also removed (Fig. $8 E$ ). These findings demonstrate how multiple guidance receptors and cues interact to determine the axonal projection choices in the developing vertebrate forebrain. 

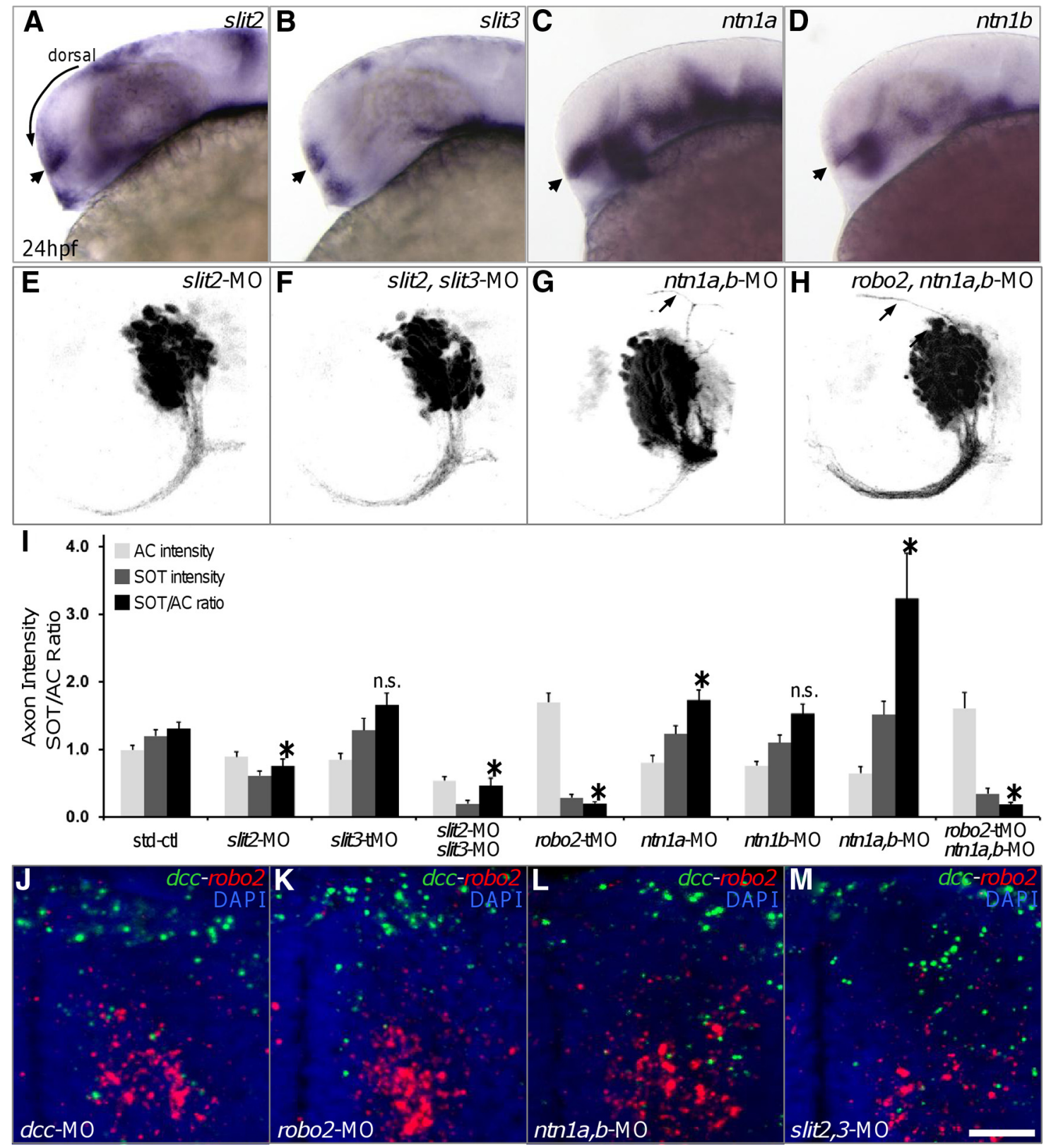

Figure 7. Inhibition of Slit or Netrin function affects the SOT or the AC formation. A-D, Expression patterns of Slits and Netrins. The probes used for whole-mount in situ hybridization are listed in the top right corner of each panel (animals mounted in lateral view, rostral to the left). The arrow in $\boldsymbol{A}$ labels the dorsal-to-ventral axis in the lateral mount view. The short arrow in each panel indicates the position of the ventral telencephalon. $\boldsymbol{E}, \boldsymbol{F}$, The SOT is reduced in slitz-MO-injected ( $\boldsymbol{E})$ or slit2-MO- and slit3-M0-injected $(\boldsymbol{F})$ embryos. $\mathbf{G}$, The AC is reduced in ntn 1a,b-M0-injected embryos. $\boldsymbol{H}$, Robo2 morpholino knockdown is epistatic to the Netrin $1 \mathrm{a}, \mathrm{b}$ morpholino knockdown. The arrows in $\boldsymbol{G}$ and $\boldsymbol{H}$ mark aberrant axons located in the dorsal telencephalon. $\boldsymbol{I}, \mathbf{Q u a n t i f i c a t i o n}$ of the AC, the SOT, and the SOT/AC ratios. Axon intensity values are presented in arbitrary units. Error bars indicate SEs. Numbers of animals analyzed for each treatment group are $n=42,33,18$, $25,36,28$, and 36 , respectively. ${ }^{*} p<0.05$ versus standard controls by Student's $t$ test. n.S., Not significant, $p>0.05$ versus standard controls. J-M, Morpholino knockdowns do not affect the expression patterns of $d c c$ and robo2 in the ADt region (frontal view, dorsal to the top). Scale bar: (in $\boldsymbol{M}) \boldsymbol{A}-\boldsymbol{D}, 150 \mu \mathrm{m} ; \boldsymbol{E}-\boldsymbol{H}, 50 \mu \mathrm{m} ; J-M, 20 \mu \mathrm{m}$.

Axonal projection patterns of ADt neurons are heterogeneous Our studies demonstrate that ADt neuronal axons choose specific projection paths at the intersection between the $\mathrm{AC}$ and the SOT (Fig. 1). These results suggest that ADt neurons are composed of distinct population of neurons based on their axon projection patterns. In adult rat, the AC is a major commissural projection, and it connects bilateral olfactory structures and basal telencephalic regions including the piriform cortex and amygadala (Jouandet and Hartenstein, 1983). The AC similarly connects the two telencephalic hemispheres in adult fish (Meek and Nieuwenhuys, 1998; Corrêa et al., 1998). The SOT likely connects the olfactory bulb and the nucleus posterior tuberis in adult fish, and it may be a precursor of the lateral forebrain bundle system (Meek and Nieuwenhuys, 1998). Currently it is not known what neuronal populations the ADt neurons may give rise to in the adult fish. Further studies are needed to determine what distinct functional role the ADt neurons may have.

Robo2 and Dcc signaling pathways are differentially required for the axonal pathfinding within the AC and the SOT

Because the ADt axon growth cones are exposed to the same extrinsic environment at the intersection between the AC and the SOT, the specific axonal projection choices of the ADt neurons (Fig. 2) are likely due to cell intrinsic differences among the $\mathrm{ADt}$ 
A

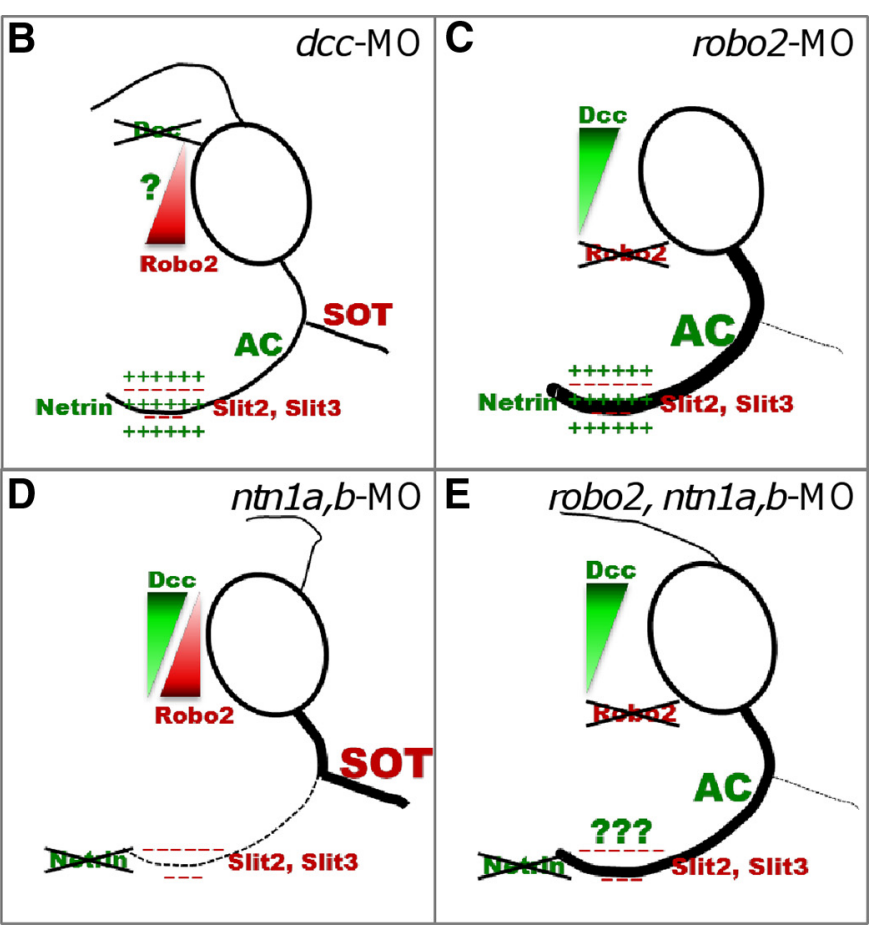

Figure 8. Schematic view of intrinsic factors and extrinsic cues that coordinate the ADt neuronal axon pathfinding in the AC and the SOT. $A$, Our data indicate Dcc and Rob02 are differentially expressed in the ADt neurons along the dorsal-ventral axis of the dorsal telencephalon. Robo2 responds to the repellent Slit signals and suppresses the attractive Netrin signals, allowing the more ventrally located ADt neurons to extend axons along the SOT pathway. $\boldsymbol{B}-\boldsymbol{E}$, Summary of functional roles of the intrinsic factors and extrinsic cues. $\boldsymbol{B}$, In the absence of Dcc, some of the ADt neurons project axons dorsally or fail to extend axons into the $\mathrm{AC}$ and the SOT. The rest of the ADt neurons project axons normally into the $\mathrm{AC}$ and the SOT, and likely use other guidance receptors (question mark) to sense the attractive cues at the AC. $C$, In the absence of Rob02, the ADt axons are no longer repelled by the Slits, and most ADt axons are attracted by the Netrin 1 cue and extend along the AC. $\boldsymbol{D}$, Removal of the attractive Netrin 1 cue causes ADt neurons to extend axons along the SOT pathway. Some ADt neurons also extend axons in the dorsal telencephalon regions. $E$, Removal of the Rob02 function is epistatic to the removal of the Netrin 1 function in terms of the SOT/AC projection pathway choices. In the absence of both the Rob02 and Netrin 1 functions, the ADt neurons fail to project axons into the SOT. These ADt neuronal axons that extend along the AC likely respond to attractive cues (question mark) other than Netrin1.

neurons. Our results show that the guidance receptors Dcc and Robo 2 are respectively enriched in the dorsal region and the ventral region in the dorsal telencephalon, and the ADt axon projections correlate with the Dcc and Robo2 expression profiles (Fig. $3)$. Together these results suggest that Dcc and Robo2 may regulate the ADt axonal projection patterns.

The Dcc-Netrin signaling pathway is known to mediate attraction of the axonal growth cones. In zebrafish, inhibition of Netrin or Dcc functions causes some olfactory sensory axons to project aberrantly and away from netrin-expressing regions (Lakhina et al., 2012). Previous studies in knock-out mice showed that DCC is required for the guidance of commissural axons to the midline (Fazeli et al., 1997). Our results show that knocking down Dcc function causes some of the labeled ADt axons to grow into aberrant dorsal positions or to stall at the $\mathrm{AC}$ and the SOT junction (Fig. $4 E-H$ ), while in the rest of labeled ADt neurons, Dcc knockdown does not prevent formation of the $\mathrm{AC}$ or the SOT (Fig. $4 I, J$ ). These results suggest that Dcc is required in the ADt neurons before the AC-SOT guidance selection choice. Formation of aberrant axons in the forebrain regions in Dcc morpholino-injected embryos has been reported previously (Gaudin et al., 2012). Examination of the aberrant dorsal ADt axons suggests that Dcc is likely involved in the asymmetric outgrowth of the ADt neurons (Gao et al. 2012). Previous studies have implicated other guidance factors such as neuropilins and semaphorin in the guidance of the anterior commissural axon to the midline (Wolman et al., 2004). These and other factors such as Neogenin, a guidance receptor related to Dcc (Wilson and Key, 2007; De Vries and Cooper, 2008), may partially compensate for the loss of the Dcc function to mediate the AC formation.
In contrast to the Dcc knockdowns, inhibition of Robo2 function abolishes the SOT originating from the ADt neurons (Fig. $5 A-F)$. In addition, the Kaede-labeled AC tracts appear enhanced (Fig. $5 J$ ). Consistent with the group labeling by photoconversion of Kaede, single-cell-labeling results show that most ADt neurons project axons into the AC when Robo2 function is inhibited (Fig. 6). These results suggest that in the absence of Robo2 function, those SOT-projecting ADt axons extend into the AC projection pathway instead.

The Robo-Slit signaling has a conserved role in midline guidance (Ypsilanti et al., 2010). Previous studies showed that Robo proteins are highly expressed in the ipsilateral axons and prevent them from crossing the midline of Drosophila (Seeger et al., 1993; Kidd et al., 1998; Rajagopalan et al., 2000). A previous study showed that in Robol and Robo2 double knock-out mice, axons from the internal capsule fail to avoid approaching the midline (López-Bendito et al., 2007). Similarly, in Slit1 and Slit2 double knock-out mice, the corticofugal projections abnormally approach the midline and cross it (Bagri et al., 2002). In the Slit1 and Slit2 double knock-out or other compound Slit knock-out mice (Bagri et al., 2002; Unni et al., 2012), the Robo1 knock-out mice (Andrews et al., 2006), and the Robol and Robo2 double knockout mice (López-Bendito et al., 2007), the corpus callosum is reduced, and some of the corticocortical axons are directed ventrally. In zebrafish, Robo2 functions to prevent and correct RGC axon pathfinding errors before and after the axon midline crossing (Fricke et al., 2001; Hutson and Chien, 2002). Reducing Slit functions causes defasciculation of the postoptic commissure (Barresi et al., 2005). We show that inhibition of Slit2 and Slit3 function in zebrafish has similar effects as inhi- 
bition of Robo2 function, and it reduces the SOT originating from the ADt neurons (Fig. $7 E, F$ ). Thus, our results are consistent with these previous results and suggest that Robo-Slits are essential for the axons to project along ipsilateral pathways in vertebrate forebrains.

Previous reports have implicated Slitla as the ligand for the Robo2 receptor in the positioning of the longitudinal axons (Devine and Key, 2008) and in the arborization and synaptogenesis of the retinal ganglion cell axons (Campbell et al., 2007). Our studies show that Slitla is not involved in the SOT formation. These results are likely due to particular combinations of intrinsic receptors and extracellular cues experienced by different types of developing neurons.

\section{Interaction between the Robo2-Slit and the Dcc-Netrin1 signaling pathways}

Our mechanistic studies of the ADt neuronal axon development show that Robo2-Slit2/Slit3-mediated repulsion is required for the SOT formation, while the Netrin1 mediate attraction is required for the AC formation. These results suggest that Robo2 responds to the repellent Slit signals and suppresses the attractive Netrin signal (Fig. 8A). Our data support this model, as overexpression of Robo2 is able to reduce the AC and appears to enhance the SOT originating from the ADt neurons (Fig. 5I,J). Thus, the competitive actions between Slits and Netrin1 are interpreted by ADt neurons expressing different levels of Robo2, Dcc, and likely other guidance receptors, allowing the appropriate guidance choices of the $\mathrm{ADt}$ neuronal axons based upon their somata positions within the dorsal telencephalon. Interestingly, our studies also show that in the absence of the attractive Netrin 1 signaling, the ADt neurons can extend axons into the AC pathway only if the repellent Robo2 signaling is also removed (Fig. 7G, $H$ ). This result suggests that other attractive guidance cues exist in the ventral telencephalon. It also highlights the requirement of $\mathrm{Ne}$ trin 1 function in the presence of the Robo2-mediated repulsion. Finally, it is interesting to note that a previous study showed that Dcc promotes ventral growth of zebrafish spinal cord commissural primary ascending neuron axons through inhibition of Robo2 (Bonner et al., 2012). Therefore, the interaction between the Robo2-Slit and the Dcc-Netrin1 signaling pathways may play roles in a variety of guidance choice mechanisms in the CNS.

\section{Intrinsic factors and the specification of the ADt neurons}

Our studies focused on the function of the guidance factors that act directly on the development of axon projections. Multiple transcription factors and guidance receptors regulate the specification and axonal pathfinding of the diverse neuronal populations (Polleux et al., 2007; Molyneaux et al., 2007). Previous studies showed that the axonal tracts in zebrafish and mouse brain were perturbed when the expression of the transcription factor, such as Pax6 (Mastick et al., 1997) or Pax2 (Macdonald et al., 1997), was disrupted. A previous study showed that the transcription factors Lhx2 and Lhx9 regulate directly the expression of the receptor Rig- 1 to mediate the guidance of the dllc axons to cross the midline in mouse spinal cord (Wilson et al., 2008). It has also been reported that Nkx2.9 may regulate Robo2 expression in the spinal accessory motor neurons (SACMNs) for the SACMN axon exit from the spinal cord (Bravo-Ambrosio et al., 2012). It should be interesting to examine how transcription factors may regulate the spatial and temporal distribution of Robo2 and other guidance receptors in the anterior dorsal telencephalon.

\section{References}

Anderson RB, Key B (1996) Expression of a novel N-CAM glycoform (NOC-1) on axon tracts in embryonic Xenopus brain. Dev Dyn 207:263-269.

Ando R, Hama H, Yamamoto-Hino M, Mizuno H, Miyawaki A (2002) An optical marker based on the UV-induced green-to-red photoconversion of a fluorescent protein. Proc Natl Acad Sci U S A 99:12651-12656.

Andrews W, Liapi A, Plachez C, Camurri L, Zhang J, Mori S, Murakami F, Parnavelas JG, Sundaresan V, Richards LJ (2006) Robol regulates the development of major axon tracts and interneuron migration in the forebrain. Development 133:2243-2252.

Asakawa K, Suster ML, Mizusawa K, Nagayoshi S, Kotani T, Urasaki A, Kishimoto Y, Hibi M, Kawakami K (2008) Genetic dissection of neural circuits by Tol2 transposon-mediated Gal4 gene and enhancer trapping in zebrafish. Proc Natl Acad Sci U S A 105:1255-1260.

Bagri A, Marín O, Plump AS, Mak J, Pleasure SJ, Rubenstein JL, TessierLavigne M (2002) Slit proteins prevent midline crossing and determine the dorsoventral position of major axonal pathways in the mammalian forebrain. Neuron 33:233-248.

Barresi MJF, Hutson LD, Chien C-B, Karlstrom RO (2005) Hedgehog regulated Slit expression determines commissure and glial cell position in the zebrafish forebrain. Development 132:3643-3656.

Bonner J, Letko M, Nikolaus OB, Krug L, Cooper A, Chadwick B, Conklin P, Lim A, Chien CB, Dorsky RI (2012) Midline crossing is not required for subsequent pathfinding decisions in commissural neurons. Neural Dev 7:18.

Bravo-Ambrosio A, Mastick G, Kaprielian Z (2012) Motor axon exit from the mammalian spinal cord is controlled by the homeodomain protein Nkx2.9 via Robo-Slit signaling. Development 139:1435-1446.

Campbell DS, Stringham SA, Timm A, Xiao T, Law MY, Baier H, Nonet ML, Chien CB (2007) Slitla inhibits retinal ganglion cell arborization and synaptogenesis via Robo2-dependent and -independent pathways. Neuron 55:231-245.

Chédotal A, Richards LJ (2010) Wiring the brain: the biology of neuronal guidance. Cold Spring Harb Perspect Biol 2:a001917.

Chitnis AB, Kuwada JY (1990) Axonogenesis in the brain of zebrafish embryos. J Neurosci 10:1892-1905.

Chitnis AB, Kuwada JY (1991) Elimination of a brain tract increases errors in pathfinding by follower growth cones in the zebrafish embryo. Neuron 7:277-285.

Corrêa SA, Grant K, Hoffmann A (1998) Afferent and efferent connections of the dorsocentral telencephalon in an electrosensory teleost, Gymnotus carapo. Brain Behav Evol 52:81-98.

Devine CA, Key B (2008) Robo-Slit interactions regulate longitudinal axon pathfinding in the embryonic vertebrate brain. Dev Biol 313:371-383.

De Vries M, Cooper HM (2008) Emerging roles for neogenin and its ligands in CNS development. J Neurochem 106:1483-1492.

Easter SS Jr, Ross LS, Frankfurter A (1993) Initial tract formation in the mouse brain. J Neurosci 13:285-299.

Easter SS Jr, Burrill J, Marcus RC, Ross LS, Taylor JS, Wilson SW (1994) Initial tract formation in the vertebrate brain. Prog Brain Res 102:79-93.

Fazeli A, Dickinson SL, Hermiston ML, Tighe RV, Steen RG, Small CG, Stoeckli ET, Keino-Masu K, Masu M, Rayburn H, Simons J, Bronson RT, Gordon JI, Tessier-Lavigne M, Weinberg RA (1997) Phenotype of mice lacking functional Deleted in colorectal cancer (Dcc) gene. Nature 386:796-804.

Fricke C, Chien CB (2005) Cloning of full-length zebrafish dcc and expression analysis during embryonic and early larval development. Dev Dyn 234:732-739.

Fricke C, Lee JS, Geiger-Rudolph S, Bonhoeffer F, Chien CB (2001) astray, a zebrafish roundabout homolog required for retinal axon guidance. Science (New York) 292:507-510.

Gao J, Zhang C, Yang B, Sun L, Zhang C, Westerfield M, Peng G (2012) Dcc regulates asymmetric outgrowth of forebrain neurons in zebrafish. PloS One 7:e36516.

Gaudin A, Hofmeister W, Key B (2012) Chemoattractant axon guidance cues regulate de novo axon trajectories in the embryonic forebrain of zebrafish. Dev Biol 367:126-139.

Hutson LD, Chien CB (2002) Pathfinding and error correction by retinal axons: the role of astray/robo2. Neuron 33:205-217.

Hutson LD, Jurynec MJ, Yeo SY, Okamoto H, Chien CB (2003) Two divergent slit1 genes in zebrafish. Dev Dyn 228:358-369. 
Jouandet ML, Hartenstein V (1983) Basal telencephalic origins of the anterior commissure of the rat. Exp Brain Res 50:183-192.

Kanki JP, Kuwada JY (2000) Growth cones utilize both widespread and local directional cues in the zebrafish brain. Dev Biol 219:364-372.

Kastenhuber E, Kern U, Bonkowsky JL, Chien CB, Driever W, Schweitzer J (2009) Netrin-DCC, Robo-Slit, and heparan sulfate proteoglycans coordinate lateral positioning of longitudinal dopaminergic diencephalospinal axons. J Neurosci 29:8914-8926.

Kidd T, Brose K, Mitchell KJ, Fetter RD, Tessier-Lavigne M, Goodman CS, Tear G (1998) Roundabout controls axon crossing of the CNS midline and defines a novel subfamily of evolutionarily conserved guidance receptors. Cell 92:205-215.

Kimmel CB, Ballard WW, Kimmel SR, Ullmann B, Schilling TF (1995) Stages of embryonic development of the zebrafish. Dev Dyn 203:253-310.

Klein A, Kroon DJ, Hoogeveen Y, Kool LJ, Renema WK, Slump CH (2011) Multimodal image registration by edge attraction and regularization using a B-spline grid. Proc SPIE 7962:796220.

Lakhina V, Marcaccio CL, Shao X, Lush ME, Jain RA, Fujimoto E, Bonkowsky JL, Granato M, Raper JA (2012) Netrin/DCC signaling guides olfactory sensory axons to their correct location in the olfactory bulb. J Neurosci 32:4440-4456.

Lauderdale JD, Davis NM, Kuwada JY (1997) Axon tracts correlate with netrin-1a expression in the zebrafish embryo. Mol Cell Neurosci 9:293-313.

Lee JS, Ray R, Chien CB (2001) Cloning and expression of three zebrafish roundabout homologs suggest roles in axon guidance and cell migration. Dev Dyn 221:216-230.

López-Bendito G, Flames N, Ma L, Fouquet C, Di Meglio T, Chedotal A, Tessier-Lavigne M, Marín O (2007) Robo1 and Robo2 cooperate to control the guidance of major axonal tracts in the mammalian forebrain. J Neurosci 27:3395-3407.

Macdonald R, Scholes J, Strähle U, Brennan C, Holder N, Brand M, Wilson SW (1997) The Pax protein Noi is required for commissural axon pathway formation in the rostral forebrain. Development 124:2397-2408.

Mastick GS, Davis NM, Andrew GL, Easter SS (1997) Pax-6 functions in boundary formation and axon guidance in the embryonic mouse forebrain. Development 124:1985-1997.

Meek J, Nieuwenhuys R (1998) Holosteans and teleosts. In: The central nervous system of vertebrates, Vol 1. (Nieuwenhuys R, Donkelaar HJ, Nicholson C, eds), pp 759-938. Heidelberg: Springer.

Miyasaka N, Morimoto K, Tsubokawa T, Higashijima S, Okamoto H, Yoshihara Y (2009) From the olfactory bulb to higher brain centers: genetic visualization of secondary olfactory pathways in zebrafish. J Neurosci 29:4756-4767.

Molyneaux BJ, Arlotta P, Menezes JRL, Macklis JD (2007) Neuronal subtype specification in the cerebral cortex. Nat Rev Neurosci 8:427-437.

Park KW, Urness LD, Senchuk MM, Colvin CJ, Wythe JD, Chien CB, Li DY (2005) Identification of new netrin family members in zebrafish: developmental expression of netrin 2 and netrin 4. Dev Dyn 234:726-731.

Patel CK, Rodriguez LC, Kuwada JY (1994) Axonal outgrowth within the abnormal scaffold of brain tracts in a zebrafish mutant. J Neurobiol 25:345-360.

Peng G, Westerfield M (2006) Lhx5 promotes forebrain development and activates transcription of secreted Wnt antagonists. Development 133:3191-3200.
Polleux F, Ince-Dunn G, Ghosh A (2007) Transcriptional regulation of vertebrate axon guidance and synapse formation. Nat Rev Neurosci 8:331-340.

Rajagopalan S, Nicolas E, Vivancos V, Berger J, Dickson BJ (2000) Crossing the midline: roles and regulation of Robo receptors. Neuron 28:767-777.

Seeger M, Tear G, Ferres-Marco D, Goodman CS (1993) Mutations affecting growth cone guidance in Drosophila: genes necessary for guidance toward or away from the midline. Neuron 10:409-426.

Strähle U, Fischer N, Blader P (1997) Expression and regulation of a netrin homologue in the zebrafish embryo. Mech Dev 62:147-160.

Suli A, Mortimer N, Shepherd I, Chien CB (2006) Netrin/DCC signaling controls contralateral dendrites of octavolateralis efferent neurons. J Neurosci 26:13328-13337.

Talbot JC, Johnson SL, Kimmel CB (2010) hand2 and Dlx genes specify dorsal, intermediate and ventral domains within zebrafish pharyngeal arches. Development 137:2507-2517.

Unni DK, Piper M, Moldrich RX, Gobius I, Liu S, Fothergill T, Donahoo AL, Baisden JM, Cooper HM, Richards LJ (2012) Multiple Slits regulate the development of midline glial populations and the corpus callosum. Dev Biol 365:36-49.

Viktorin G, Chiuchitu C, Rissler M, Varga ZM, Westerfield M (2009) Emx3 is required for the differentiation of dorsal telencephalic neurons. Dev Dyn 238:1984-1998.

Westerfield M (2007) The zebrafish book: a guide for the laboratory use of zebrafish Danio rerio, Ed 5. Eugene, OR: Oregon UP.

Westerfield M, Wegner J, Jegalian BG, DeRobertis EM, Püschel AW (1992) Specific activation of mammalian Hox promoters in mosaic transgenic zebrafish. Genes Dev 6:591-598.

Wilson NH, Key B (2006) Neogenin interacts with RGMa and netrin-1 to guide axons within the embryonic vertebrate forebrain. Dev Biol 296:485-498

Wilson NH, Key B (2007) Neogenin: one receptor, many functions. Int J Biochem Cell Biol 39:874-878.

Wilson SI, Shafer B, Lee KJ, Dodd J (2008) A molecular program for contralateral trajectory: Rig-1 control by LIM homeodomain transcription factors. Neuron 59:413-424.

Wilson SW, Ross LS, Parrett T, Easter SS (1990) The development of a simple scaffold of axon tracts in the brain of the embryonic zebrafish, Brachydanio rerio. Development 108:121-145.

Wolman MA, Liu Y, Tawarayama H, Shoji W, Halloran MC (2004) Repulsion and attraction of axons by semaphorin3D are mediated by different neuropilins in vivo. J Neurosci 24:8428-8435.

Wyatt C, Ebert A, Reimer MM, Rasband K, Hardy M, Chien CB, Becker T, Becker CG (2010) Analysis of the astray/robo2 zebrafish mutant reveals that degenerating tracts do not provide strong guidance cues for regenerating optic axons. J Neurosci 30:13838-13849.

Yeo SY, Little MH, Yamada T, Miyashita T, Halloran MC, Kuwada JY, Huh TL, Okamoto H (2001) Overexpression of a slit homologue impairs convergent extension of the mesoderm and causes cyclopia in embryonic zebrafish. Dev Biol 230:1-17.

Ypsilanti AR, Zagar Y, Chédotal A (2010) Moving away from the midline: new developments for Slit and Robo. Development 137:1939-1952.

Zolessi FR, Poggi L, Wilkinson CJ, Chien CB, Harris WA (2006) Polarization and orientation of retinal ganglion cells in vivo. Neural Dev 1:2. 